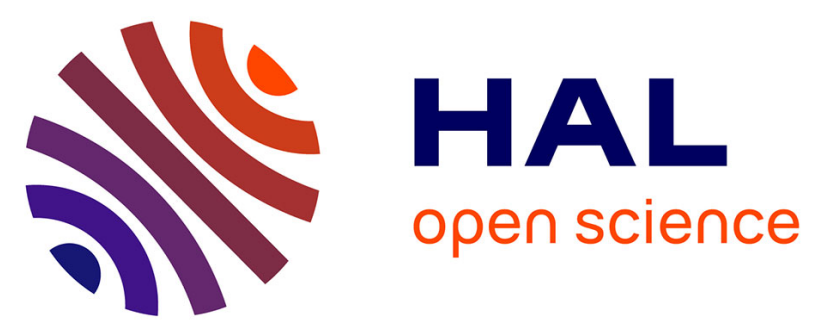

\title{
Quantifying the effect of two-point correlations on the effective elasticity of specific classes of random porous materials with and without connectivity
}

Othmane Zerhouni, Sébastien Brisard, Kostas Danas

\section{- To cite this version:}

Othmane Zerhouni, Sébastien Brisard, Kostas Danas. Quantifying the effect of two-point correlations on the effective elasticity of specific classes of random porous materials with and without connectivity. International Journal of Engineering Science, 2021, 166, pp.103520. 10.1016/j.ijengsci.2021.103520 . hal-03275328

\section{HAL Id: hal-03275328 \\ https: / hal-enpc.archives-ouvertes.fr/hal-03275328}

Submitted on 1 Jul 2021

HAL is a multi-disciplinary open access archive for the deposit and dissemination of scientific research documents, whether they are published or not. The documents may come from teaching and research institutions in France or abroad, or from public or private research centers.
L'archive ouverte pluridisciplinaire HAL, est destinée au dépôt et à la diffusion de documents scientifiques de niveau recherche, publiés ou non, émanant des établissements d'enseignement et de recherche français ou étrangers, des laboratoires publics ou privés.

\section{(1) (1) $\$$}

Distributed under a Creative Commons Attribution - NonCommercial - NoDerivatives 44.0 


\title{
Quantifying the effect of two-point correlations on the effective elasticity of specific classes of random porous materials with and without connectivity
}

\author{
O. Zerhouni, S. Brisard, K. Danas
}

This is the accepted version of the following article: "Quantifying the effect of two-point correlations on the effective elasticity of specific classes of random porous materials with and without connectivity", which has been published in final form at https://doi.org/10.1016/j.ijengsci.2021.103520.

(C) 2021. This manuscript version is made available under the CC-BY-NC-ND 4.0 license. See Elsevier Sharing Policy. 


\title{
Quantifying the effect of two-point correlations on the effective elasticity of specific classes of random porous materials with and without connectivity
}

\author{
O. Zerhouni ${ }^{\mathrm{a}, \mathrm{b}}$, S. Brisard ${ }^{\mathrm{b}, *}$, K. Danas ${ }^{\mathrm{a}, *}$ \\ ${ }^{a}$ LMS, CNRS, École Polytechnique, Institut Polytechnique de Paris, F-91128 Palaiseau, France \\ ${ }^{b}$ Navier, Ecole des Ponts, Univ Gustave Eiffel, IFSTTAR, CNRS, F-77455 Marne-la-Vallée, France
}

\begin{abstract}
It is well-known by now that the Hashin-Shtrikman bounds imply that the two-point correlation functions are not in general sufficient to estimate accurately the response of composites, especially when their underlying phases exhibit infinite contrast, e.g., porous materials. Starting from this longstanding, albeit qualitative result, this work investigates quantitatively the relevance of using two-point correlations to model the effective elastic properties of specific isotropic porous materials with and without connectivity. To achieve this in an unambiguous manner, we propose three different microstructures that share almost identical two-point statistics by design but are rather different morphologically. The choice of these microstructures is driven by their wide use in several practical problems ranging from polymers to geomaterials. The first microstructure is obtained by a random sequential adsorption (RSA) of non-overlapping, polydisperse, spherical and ellipsoidal voids oriented randomly in a unit-cell. The second one, termed connected random sequential adsorption (CRSA), is obtained from the first one by adding controlled connectivity via cylindrical channels of circular cross-section. The porosity resulting from connectivity is compensated by reducing the size of the existing voids to have the same overall porosity. Interestingly, we find that connectivity does not affect the corresponding two-point statistics. Finally, using as an input the numerical one- and two-point correlations of the RSA, we reconstruct a thresholded Gaussian random field (TGRF) microstructure. Using FFT numerical simulations, we show that the resulting effective elastic properties are very different for the three generated microstructures, despite them sharing nearly the same two-point correlation functions. We show, further, that the introduction of connectivity, and in particular the partial volume fraction of the connected channels, even small, affects strongly the resulting effective elasticity of the composite.
\end{abstract}

Keywords: Random porous materials, Homogenization, Effective elastic properties, Random Sequential Adsorption, Gaussian Random Fields, Connectivity, Two-point Correlations

\section{Introduction}

Random porous materials are omnipresent in nature (e.g. rocks and geomaterials) and in industry, where they are engineered to exhibit specific properties: auxetic metamaterials (Ali and Rehman, 2011; Ren et al., 2015) or highlystiff and light-weight microstructures (Zerhouni et al., 2019; Tarantino et al., 2019). The effective physical properties of such materials are strongly related to their microstructure which offers a large field of possibilities for material design (Ashby, 2013). In order to fulfill their potential, modeling the link between the microstructure and the effective properties of random materials is an important scientific and industrial challenge (Escoda et al., 2011; Dirrenberger et al., 2013; Cadiou et al., 2019).

Various mean field/effective field estimates of the effective elastic properties have been derived from the Eshelby (1957) solution to the ellipsoidal inhomogeneity problem (see Budiansky, 1965; Mori and Tanaka, 1973; Milton, 1985; Norris, 1985; Benveniste, 1987; Berryman et al., 2002; Ramtani et al., 2009, among others). These models are

\footnotetext{
${ }^{*}$ Corresponding authors.

Email addresses: othmane.zerhouni@polytechnique.edu (O. Zerhouni), sebastien.brisard@univ-eiffel.fr (S. Brisard), konstantinos.danas@polytechnique.edu (K. Danas)
} 
extremely versatile with respect to the material non-linearities and/or multi-physics couplings that they can account for.

In terms of the microstructure description, however, they are fairly limited (although more advanced models can be proposed for specific microstructures, see for example Kanaun (2016)). The simplest of all microstructural descriptors is the volume fraction or one-point probability function of the constituents (e.g. the porosity in the present case). It is a measurable quantity via tomography, density and volume measurements during fabrication, albeit tricky if several scales are involved. Anything beyond this simple microstructural description becomes increasingly complicated. Experiments may provide information on the overall symmetry of the material such as isotropy, transverse isotropy or orthotropy. In those cases, one may resort to the use of two- or three-point probability functions, directly measured from the experimental images or to approximations such as the introduction of a representative spherical or ellipsoidal pore shape and orientation with given distribution Ponte Castañeda and Willis (1995) able to reproduce in simple cases the average response but usually poor in describing the actual microstructure. Other descriptors, such as connectivity, tortuosity (Sevostianova et al., 2010) or the pore-size distribution including clustering (Torquato, 2002; Altendorf et al., 2014) are usually discarded or remain hidden in the original approximation. As an example, the classical Mori and Tanaka (1973) and Willis (1981) estimates implicitly assume an infinity of inclusion sizes.

This intrinsic limitation calls for an in-depth quantitative study of the effect of such statistical descriptors (besides porosity and pore aspect ratio) and their relative influence on the effective response of the composite material.

Specifically, a natural hierarchy of statistical descriptors of the microstructure would be the set of $n$-point probability functions (also abusively called $n$-point correlation functions). In fact, Torquato (1997) proposed for a random two-phase composite an exact expansion of the effective moduli involving the whole (infinite) set of such statistical descriptors. In practice, experimental measurement of the three-point correlation functions is difficult (Berryman, 1985; Roberts, 1997) and impractical for higher-order correlation functions. It is therefore natural to try to restrict the set of statistical descriptors of the microstructure to two-point correlation functions to predict the effective properties. Note that experimental measurement of these correlation functions is possible, through image analysis (Karsanina et al., 2015) or other techniques such as small angle scattering of X-rays or neutrons (Drummy et al., 2008; Baniassadi et al., 2011).

A popular approach to achieve this is to measure experimentally the two-point probability functions on a real sample, and generate a 3D virtual microstructure that exhibits the same two-point correlations by means of an appropriate stochastic model. Then, the effective properties of the real material can be estimated from the computation of the effective properties of the virtual microstructure. Depending on the material and physical process under consideration, this strategy delivered good results (Levitz, 1998, for molecular transport in Vycorß) or poor agreement with experimental data (Øren and Bakke, 2002, for permeability). The latter case reveals that two-point statistics is generally insufficient to deliver an accurate estimate of the effective transport properties. In some cases, this lack of information can be counterbalanced by the random process itself that might deliver microstructures which are close enough to the true microstructure without further efforts. If that is not the case, the reconstructed microstructure can be constrained by other statistical descriptors (besides the two-point correlation functions) such as chord-length distributions (Teubner, 1991), three-point descriptors (Roberts, 1997), grain-size distribution (Roubin et al., 2015) or geodesic tortuosity (Neumann et al., 2019).

To the best of our knowledge, a properly designed and comparative quantitative analysis of the information that the two-point correlation functions deliver on the effective elastic properties of a random heterogeneous material is lacking. Restricting the present study to porous materials, the question boils down to finding out whether the effective elastic properties of two heterogeneous materials that share the same two-point correlation functions are equal or at least similar. If the answer to this question is "yes", then the two-point correlation functions can be considered as "good" descriptors of the microstructure, at least for upscaling the linear elastic properties. If the answer is "no", we are interested to quantify the resulting differences especially in porous materials comprising similar but also widelyused types of microstructures.

To investigate this question, we adopt a purely numerical approach that guarantees that model discrepancies are not affected by other sources of errors (such as experimental uncertainties). We therefore generate several virtual, significantly different isotropic porous microstructures with the same two-point correlation functions and compute their effective elastic properties through full-field simulations. We emphasize that since we restrict attention in this study to isotropic effective responses, the orientation of the ellipsoidal voids in the RSA and CRSA microstructures (to be described below) is taken to be isotropic (uniformly distributed over the unit sphere) and thus is not considered 
a parameter of the problem henceforth. Interestingly, we find that the results are widely different, which confirms in an unambiguous way that the two-point correlation functions not only are "insufficient" descriptors of the microstructure, as perhaps one could infer from the early studies of Hashin-Shtrikman for infinite contrast composites, but also that the resulting differences in elastic properties are substantial even for porous materials belonging to similar classes.

At this point, it should be noted that many stochastic processes can be simulated to generate random microstructures: thresholded gaussian random fields (Teubner, 1991; Levitz, 1998) and various extensions (Roberts, 1997; Roubin et al., 2015), voxel-based simulations (Cule and Torquato, 1999), boolean models (Willot and Jeulin, 2009; Escoda et al., 2015) or particle-based methods (Donev et al., 2005a,b). We initially settled on thresholded gaussian random fields (TGRF) and random sequential addition (RSA) (Lopez-Pamies et al., 2013; Anoukou et al., 2018) to generate the two families of microstructures to be compared. In line with what was observed for e.g. conductivity (Øren and Bakke, 2002), our results showed that the effective properties of the two types of microstructures were quite different. Connectivity obviously plays a significant role on the effective transport properties of heterogeneous materials, and RSA (essentially disconnected) and TGRF (Roubin et al., 2015; Roubin and Colliat, 2016) are substantially different from the perspective of connectivity. In order to assess the effect of connectivity on the effective elastic properties, we develop a third family of microstructures, namely a connected random sequential addition (CRSA). With this third family of microstructures, we show that connectivity -even when the connecting part occupies only a minor fraction of the overall porosity- is an extremely important microstructural property affecting critically the effective elastic properties, a result which we did not expect.

The paper is organized as follows. Section 2 provides background information on second-order statistical descriptors of random microstructures. In Section 3, we describe the generation of the three types of microstructures considered in this paper, namely: random sequential addition of polydisperse spheroids (RSA), connected RSA microstructures (CRSA), and thresholded Gaussian random fields (TGRF). We note, in particular, that the algorithm that we use to generate the CRSA microstructures is new. We also discuss in Section 3 the determination of their effective elastic properties. We then show in Section 4 that connectivity, and particularly the type of connectivity, is an essential microstructural feature for the estimation of the effective elastic properties. By contrast, in Section 5, we show-by comparing the three aforementioned microstructures-that the two-point correlations constitute an insufficient microstructural descriptor for estimating the effective elastic properties.

\section{Background: second-order statistical descriptors of the microstructure}

We consider a random two-phase material: index "s" refers to the solid phase, while index "p" refers to the pore space. Such a material is fully defined by the indicator function $\chi_{\mathrm{p}}(\mathbf{x})$ of the pore space. This function is a random field with values in $\{0,1\}$, that is equal to 1 if and only if $\mathbf{x}$ belongs to the pores. The indicator function of the solid phase $\chi_{\mathrm{s}}$ is then given by $\chi_{\mathrm{s}}(\mathbf{x})=1-\chi_{\mathrm{p}}(\mathbf{x})$.

For statistically homogeneous media, the porosity $c$ (volume fraction of pores) coincides with the expectation of $\chi_{\mathrm{p}}$, defined as $c=\mathbb{E}\left[\chi_{\mathrm{p}}(\mathbf{x})\right]$, where the right-hand side does in fact not depend on $\mathbf{x}$. We further define the two-point correlation functions

$$
S_{\alpha \beta}(\mathbf{r})=\mathbb{E}\left[\chi_{\alpha}(\mathbf{x}) \chi_{\beta}(\mathbf{x}+\mathbf{r})\right] \quad \alpha, \beta \in\{\mathrm{p}, \mathrm{s}\},
$$

which gives the probability that point $\mathbf{x}$ belongs to phase $\alpha$, while point $\mathbf{x}+\mathbf{r}$ belongs to phase $\beta$. For statistically homogeneous materials, this probability does not depend on $\mathbf{x}$ and we write

$$
S_{\alpha \beta}(\mathbf{r})=\mathbb{E}\left[\chi_{\alpha}(\mathbf{0}) \chi_{\beta}(\mathbf{r})\right], \quad \alpha, \beta \in\{\mathrm{p}, \mathrm{s}\} .
$$

Note that, for isotropic materials, the correlation functions $S_{\alpha \beta}(r)$ depend on the norm $r=\|\mathbf{r}\|$ of the lag-vector $\mathbf{r}$ only. The three two-point correlation functions thus defined are in fact linearly dependent $\left(\right.$ since $\left.\chi_{\mathrm{p}}+\chi_{\mathrm{s}}=1\right)$, i.e.,

$$
S_{\mathrm{ss}}(\mathbf{r})=1-2 c+S_{\mathrm{pp}}(\mathbf{r}) \quad \text { and } \quad S_{\mathrm{ps}}(\mathbf{r})=c-S_{\mathrm{pp}}(\mathbf{r}) .
$$

In the remainder of this paper, we will therefore focus on the two-point correlation function of the pore space, $S_{\mathrm{pp}}$, which will be referred to as the two-point correlation function and denoted simply as $S$. To close this section, some elementary properties of $S$ are recalled. 
Since $\chi_{\mathrm{p}}(\mathbf{r}) \leq 1$, we have $\chi_{\mathrm{p}}(\mathbf{0}) \chi_{\mathrm{p}}(\mathbf{r}) \leq \chi_{\mathrm{p}}(\mathbf{0})$ and, taking the average yields $S(\mathbf{r}) \leq c$. We further make the assumption of no-long range order, which states that the phases at $\mathbf{x}$ and $\mathbf{x}+\mathbf{r}$ are statistically independent. In other words,

$$
S(\mathbf{r}) \rightarrow c^{2} \quad \text { when } \quad\|\mathbf{r}\| \rightarrow+\infty .
$$

For isotropic materials, Debye et al. (1957) have shown that the slope of the two-point correlation function $S(r)$ at the origin $r=0$ is related to the specific surface $S_{\mathrm{v}}$, defined as the interface area per unit volume of material. More precisely

$$
\left.\frac{\mathrm{d} S}{\mathrm{~d} r}\right|_{r=0}=-\frac{S_{\mathrm{v}}}{4} .
$$

Finally, it is observed that the Fourier transform of the correlation function $\hat{S}(\mathbf{k})=\hat{S}_{\mathrm{pp}}(\mathbf{k})$ is equal to the power spectrum of the indicator function $\chi_{\mathrm{p}}$ of the microstructure. Extraction of the two-point correlation function from a (2D/3D) image can therefore be performed efficiently by means of discrete Fourier transforms, using the fast Fourier transform (Drummy et al., 2008).

\section{Generation and mechanical simulation of the microstructures}

In this section, we describe the three families of microstructures analyzed in the present study and the numerical framework used to compute their effective elastic properties. In particular, the connected RSA microstructure is based on the non-connected RSA microstructure and is presented here for the first time.

\subsection{Generation of non-connected RSA microstructures}

We first consider assemblies of non-ovelapping, polydisperse ellipsoidal voids resulting from a random sequential addition process (RSA) as extended to polydisperse assemblies by Lopez-Pamies et al. (2013) and Anoukou et al. (2018). Each ellipsoidal void is inserted in a sequential manner. Specifically, a random center and a random orientation are proposed for the current void, whose shape is already pre-defined. If the insertion results in no overlap, the proposal is accepted and the simulation moves to the next void. In case of overlap, the proposal is declined, and a new center and orientation are drawn for the current void. Several points should be noted: $i$. centers are drawn uniformly in the cubic unit-cell $(0, L)^{3}$, while orientations are chosen isotropically (each orientation being equiprobable) ${ }^{1} ; i i$. other acceptance criteria (besides non-overlap) for discretization purposes are also added, see below; iii. the microstructure is periodic, which requires special care in asserting non-overlap (Anoukou et al., 2018).

The total porosity (volume fraction of pores) is $c$, so that the total volume occupied by the pores is $c V$, where $V=L^{3}$ is the volume of the unit-cell. The ellipsoidal pores are organized in $N_{\text {phases }}$ families (or phases); within each family $r=1, \ldots, N_{\text {phases }}$, all pores have the same radii $a_{1}^{(r)}, a_{2}^{(r)}$ and $a_{3}^{(r)}$ and random orientations (see Fig. 1). There are $N^{(r)}$ pores in phase $r$ and the total number of pores is

$$
N_{\text {pores }}=\sum_{r=1}^{N_{\text {phases }}} N^{(r)} .
$$

We further introduce the following dimensionless quantities: first, the pore aspect ratios

$$
\omega_{1}^{(r)}=\frac{a_{3}^{(r)}}{a_{1}^{(r)}} \quad \text { and } \quad \omega_{2}^{(r)}=\frac{a_{3}^{(r)}}{a_{2}^{(r)}},
$$

then the size ratio

$$
\alpha^{(r)}=\frac{a_{3}^{(r)}}{a_{3}^{(1)}},
$$

\footnotetext{
${ }^{1}$ In order to achieve an equiprobable distribution of orientations in the unit sphere and thus approach the overall isotropy more efficiently, we use three Euler angles, $\phi, \theta, \psi$. It is recalled that the angles $\phi, \psi$ are uniformly distributed in $(-\pi, \pi)$, whereas for the third one, $\cos (\theta)$ is uniformly distributed in $(-1,1)$ followed by inversion of the cosine.
} 


\begin{tabular}{c|cccccc}
\hline$r$ & 1 & 2 & 3 & 4 & 5 & 6 \\
\hline$\alpha^{(r)}$ & 1 & 0.85 & 0.75 & 0.65 & 0.55 & 0.45 \\
$\gamma^{(r)}$ & $20 \%$ & $30 \%$ & $10 \%$ & $10 \%$ & $10 \%$ & $20 \%$ \\
\hline
\end{tabular}

Table 1: The relative size $\alpha^{(r)}$ and volume fraction $\gamma^{(r)}$ of the 6 families of spheroidal pores considered in this study.

finally, the fraction $\gamma^{(r)}$ of the pore space occupied by phase $r$

$$
\gamma^{(r)}=\frac{N^{(r)} v^{(r)}}{c L^{3}}, \quad \text { with } \quad v^{(r)}=\frac{4}{3} \pi a_{1}^{(r)} a_{2}^{(r)} a_{3}^{(r)} \quad \text { and } \quad \sum_{r=1}^{N_{\text {phases }}} \gamma^{(r)}=1
$$

In the remainder of this paper, in order to reduce the number of microstructural parameters analyzed, we will only focus on spheroids (ellipsoids with two equal radii), and the third local direction will be chosen as the axis of revolution. With no possible ambiguity, we now assume that the phases are sorted by decreasing size

$$
a_{3}^{(1)} \geq a_{3}^{(2)} \geq \cdots \geq a_{3}^{\left(N_{\text {phases }}\right)},
$$

which is also the order in which the pores are inserted in the unit cell (larger pores first). This indeed allows to reach higher volume fractions.

To summarize, RSA microstructures considered in this paper are fully described by the following parameters

$$
c, N_{\text {phases }}, a_{3}^{(1)}, \omega_{1}^{(r)}, \omega_{2}^{(r)}, \alpha^{(r)}, \gamma^{(r)} \quad\left(1 \leq r \leq N_{\text {phases }}\right) .
$$

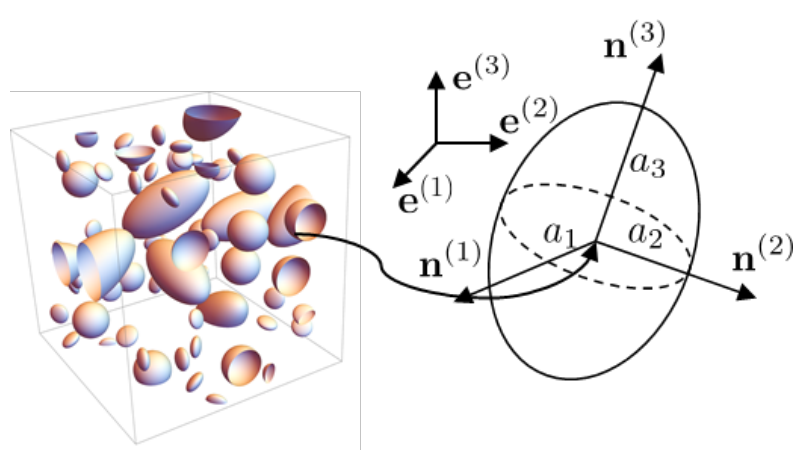

Figure 1: Example of a generated RSA microstructure, showing some of the parameters introduced in Section 3.1.

In addition, all microstructures comprise $N_{\text {phases }}=6$ phases. All pores are spheroidal with the same aspect ratio for all $N_{\text {phases }}$ families

$$
\omega_{1}^{(1)}=\omega_{2}^{(1)}=\cdots=\omega_{1}^{\left(N_{\text {phases }}\right)}=\omega_{2}^{\left(N_{\text {phases }}\right)}=\omega .
$$

The unique aspect ratio $\omega$ takes values in $\omega \in\{0.1,0.2,0.4,0.6,0.8,1\}$. The relative sizes of pores $\alpha^{(r)}$ and corresponding volume fraction $\gamma^{(r)}$ are reported in table 1 . Note that the resulting pore-size distribution is relatively large, which allows the RSA algorithm to achieve large porosities (up to 25\%). An even larger pore-size distribution may be used to reach porosities as large as $80 \%$, as detailed in Zerhouni et al. (2019) but this is beyond the scope of the present work.

Finally, the value of $a_{3}^{(1)}$ is chosen such that the microstructure contains $N^{(1)}=50$ pores of the first phase. This leads to microstructures with a total number of pores which is large enough to obtain representative effective elastic properties (Anoukou et al., 2018). With these values of the various microstructural parameters, hereafter, RSA microstructures are unambiguously described by their porosity $c$ and pore aspect ratio $\omega$ only.

We close this section by noting that the RSA algorithm presented above has been slightly biased as follows. First, the proposed location for the current ellispoid $\mathcal{E}_{i}$ is rejected if a previously inserted ellipsoid $\mathcal{E}_{j}$ is too close to $\mathcal{E}_{i}$. 
More precisely, rejection occurs if the distance between $\mathcal{E}_{i}$ and $\mathcal{E}_{j}$ is smaller than

$$
1.05 \times \frac{1}{2}\left\{\max \left[a_{1}^{\left(r_{i}\right)}, a_{2}^{\left(r_{i}\right)}, a_{3}^{\left(r_{i}\right)}\right]+\max \left[a_{1}^{\left(r_{j}\right)}, a_{2}^{\left(r_{j}\right)}, a_{3}^{\left(r_{j}\right)}\right]\right\},
$$

where $r_{i}$ and $r_{j}$ denote the phases to which $\mathcal{E}_{i}$ and $\mathcal{E}_{j}$ belong, respectively.

Second, ellipsoid $\mathcal{E}_{i}$ must not be too close to the boundary of the unit-cell. More precisely, the proposed location for ellipsoid $\mathcal{E}_{i}$ is rejected if the distance to the closest boundary is smaller than

$$
1.05 \times \max \left[a_{1}^{\left(r_{i}\right)}, a_{2}^{\left(r_{i}\right)}, a_{3}^{\left(r_{i}\right)}\right] .
$$

These two additional conditions ensure that the microstructures can be suitably discretized in a finite element framework. They are not as such necessary for discretization over a cartesian grid used in the present work (see Section 3.4). Nevertheless, these conditions lead to a discretization that no grid-cell is intersected by two ellipsoids at a time.

a)

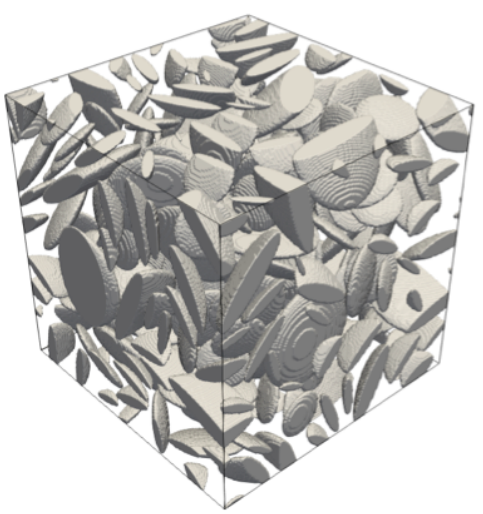

b)

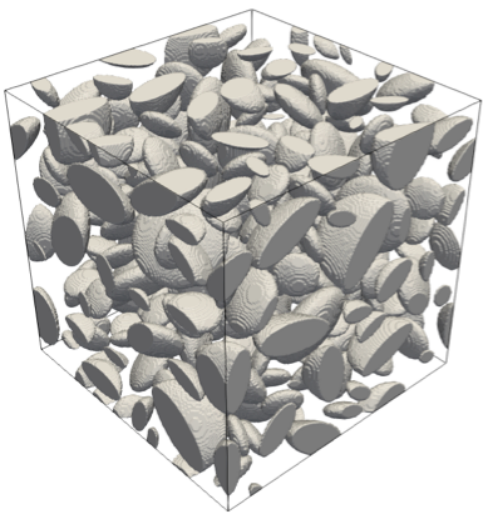

c)

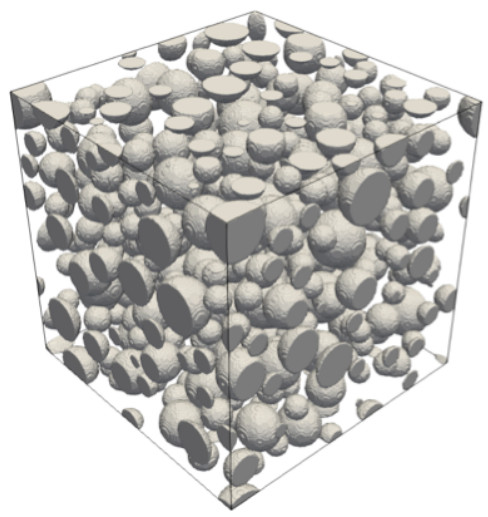

Figure 2: Examples of RSA microstructures with porosity $c=25 \%$. Unit cells containing polydisperse (multiple size) voids with aspect ratio (a) $\omega=0.2$ and $N_{\text {pores }}=204$, (b) $\omega=0.4$ and $N_{\text {pores }}=204$, (c) $\omega=1$ and $N_{\text {pores }}=408$.

\subsection{Generation of connected RSA microstructures}

The microstructures considered in this section differ from the microstructures considered in Section 3.1 by the addition of channels that connect the ellipsoidal pores. This introduces controlled connectivity in the microstructure. For this purpose, we develop a modified RSA algorithm, which is presented below. Note that the channels considered here are circular cylinders, a choice allowing to maintain the number of microstructural parameters to the minimum possible. However, the algorithm can readily be extended to more general channel shapes.

Besides the microstructural parameters listed in Eq. (11), our modified RSA algorithm is controlled by only two additional parameters, namely, $\eta$ and $\zeta$, to be defined below. For clarity, we illustrate schematically in Fig. 3 the process to obtain connectivity from the existing RSA microstructure.

The first parameter is the reduced radius $\eta$, that controls the radius $R_{i j}$ of the cylindrical channel connecting two ellipsoidal pores $\mathcal{E}_{i}$ and $\mathcal{E}_{j}$

$$
R_{i j}=\eta \min \left[a_{1}^{\left(r_{i}\right)}, a_{2}^{\left(r_{i}\right)}, a_{3}^{\left(r_{i}\right)}, a_{1}^{\left(r_{j}\right)}, a_{2}^{\left(r_{j}\right)}, a_{3}^{\left(r_{j}\right)}\right],
$$

where $r_{i}$ and $r_{j}$ are defined as in Eq. (13).

The second parameter is the coordination number $\zeta$, that controls the total number of channels in the microstructure, $N_{\text {channels }}$, as follows

$$
N_{\text {channels }}=\frac{1}{2} \zeta N_{\text {pores }}
$$

where it is recalled that $N_{\text {pores }}$ denotes the total number of pores [see Eq. (6)]. In other words, $\zeta$ is the average number of channels per pore. 


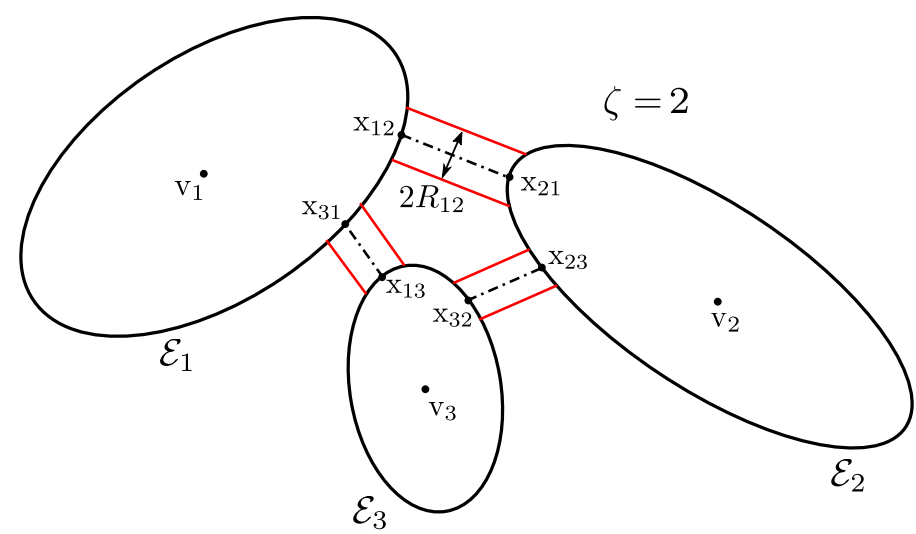

Figure 3: Two-dimensional sketch illustrating the process to obtain the CRSA microstructures. The red channels indicate the connected part of the microstructure. In this example, we have a coordination number $\zeta=2$.

The channels are introduced a posteriori in a previously generated (unconnected) RSA microstructure. The procedure relies heavily on the minimal distance between ellipsoidal pores. More precisely, for two ellipsoids $\mathcal{E}_{i}$ and $\mathcal{E}_{j}$, we define the points $\mathbf{x}_{i j} \in \mathcal{E}_{i}$ and $\mathbf{x}_{j i} \in \mathcal{E}_{j}$ that minimize

$$
\left(\mathbf{x}_{i j}, \mathbf{x}_{j i}\right)=\arg \min \left\{\left\|\mathbf{x}_{j}-\mathbf{x}_{i}\right\|,\left(\mathbf{x}_{i}, \mathbf{x}_{j}\right) \in \mathcal{E}_{i} \times \mathcal{E}_{j}\right\}
$$

as well as the actual pore-to-pore distance

$$
d_{i j}=d_{j i}=\left\|\mathbf{x}_{j i}-\mathbf{x}_{i j}\right\| .
$$

The points $\mathbf{x}_{i j}$ and $\mathbf{x}_{j i}$ and distance $d_{i j}$ are first computed using the local ball approximation described in Anoukou et al. (2018). Note that, owing to the periodicity of the simulation box, periodic images of the pores must also be considered for the determination of the above quantities. All $(i, j)$ pairs of ellipsoids are then sorted by order of increasing distance $d_{i j}$. The $N_{\text {channels }}$ first pairs are then selected, where $N_{\text {channels }}$ is given by Eq. (16). For each selected $(i, j)$ pair, a cylindrical channel is inserted. Its radius $R_{i j}$ is given by Eq. (15), while its axis is given by $\mathbf{x}_{j i}-\mathbf{x}_{i j}$. Note that this procedure may fail if the number of channels to be inserted is too large (not enough pairs of neighbouring pores available). In the microstructures presented here, the values of $\zeta$ are chosen small enough for this situation never to occur. Examples of generated CRSA microstructures are displayed in Fig. 4.

a)

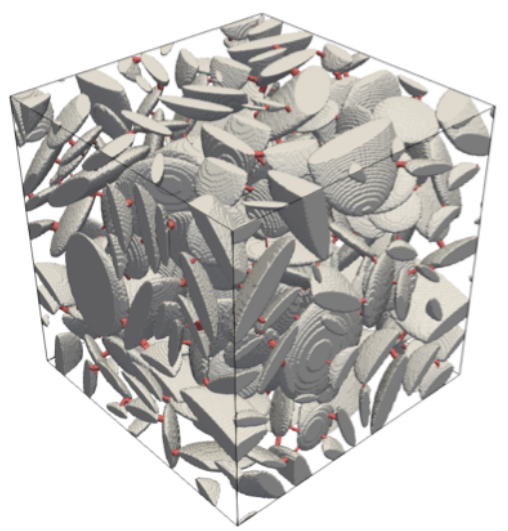

b)

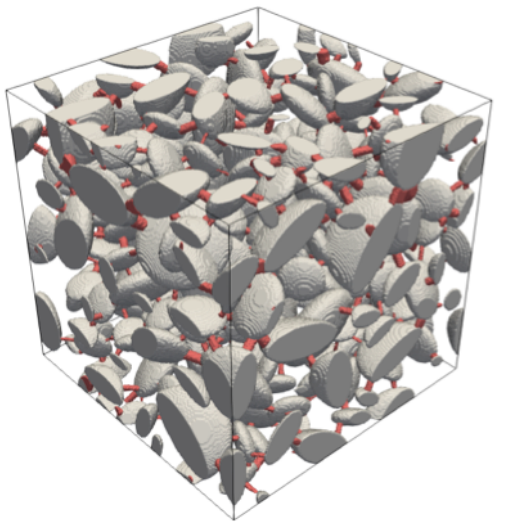

c)

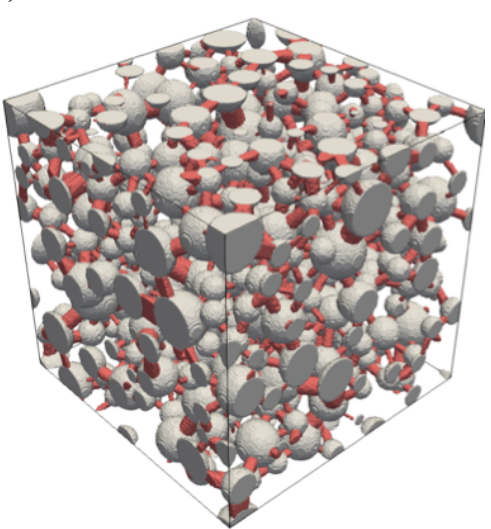

Figure 4: Examples of CRSA microstructures with porosity $c=25 \%$, channel size determined by $\eta=0.3$ and coordination number $\zeta=6$. In addition to the connecting channels the unit cells contain polydisperse (multiple size) voids with aspect ratio (a) $\omega=0.2$ and $N_{\text {pores }}=204$ (b) $\omega=0.4$ and $N_{\text {pores }}=204$ and (c) $\omega=1$ and $N_{\text {pores }}=408$. The pore channels are shown in red and constitute only a small fraction of the overall porosity. 
We close this section by further noting that introducing cylindrical channels into the RSA microstructures alters its porosity $c$ slightly. Our goal is to ensure that the final porosity of the CRSA microstructures is indeed $c$, up to an acceptable negligible error resulting from polydispersity and the random procedure itself. To enforce this requirement, the initial RSA microstructures (prior to channel insertion) were generated with a slightly lower porosity, $\tilde{c}$, the value of which was found by trial-and-error. On average, the actual porosity of the generated CRSA microstructures was within $0.1 \%$ of the prescribed value. We will show in Section 5.1, that the introduction of connectivity does not alter in essence the two-point correlation functions in the RSA microstructures.

\subsection{Generation of thresholded GRF microstructures}

The third type of microstructures considered here results from thresholding a Gaussian random field (TGRF microstructures). The main reason for this choice is the fact that such microstructures can be generated entirely by prescribing the two-point correlation functions. In order to investigate the relative effect of the two-point correlation functions to the effective properties, we generate the TGRF microstructures such that they exhibit the same two-point correlations as the RSA microstructures presented in Sections 3.1 (see Section 5.1). Note that using thresholded Gaussian random fields to model microstructures is a fairly well-known technique (Roberts and Teubner, 1995; Roberts, 1997; Roubin et al., 2015; Khristenko et al., 2020); it has recently been applied by Kumar et al. (2020) to the inverse design of spinodoid metamaterials. However, most works generally rely on analytical expressions of the two-point correlation functions. In the present work, we use correlation functions of real (RSA) microstructures, which are computed numerically. This opens the possibility for using this reconstruction process in any type of numerically evaluated two-point correlations.

The TGRF microstructures are defined as random fields. More precisely, the indicator function $\chi_{\mathrm{p}}(\mathbf{x})$ of the pore space is defined as

$$
\chi_{\mathrm{p}}(\mathbf{x})= \begin{cases}0 & \text { if } Y(\mathbf{x})<\lambda, \\ 1 & \text { if } Y(\mathbf{x}) \geq \lambda,\end{cases}
$$

where $\lambda$ is a prescribed threshold and $Y$ is a Gaussian random field with zero mean and unit variance

$$
\mathbb{E}[Y(\mathbf{x})]=0, \quad \mathbb{E}\left[Y^{2}(\mathbf{x})\right]=1 \quad \text { and } \quad \mathbb{E}[f(Y(\mathbf{x}))]=\frac{1}{\sqrt{2 \pi}} \int_{-\infty}^{+\infty} f(y) \exp \left(-y^{2} / 2\right) \mathrm{d} y .
$$

The value of the threshold $\lambda$ is directly related to the porosity $c$ of the microstructure to be generated. Indeed, from the above equation

$$
c=\mathbb{E}\left[\chi_{\mathrm{p}}\right]=\frac{1}{\sqrt{2 \pi}} \int_{\lambda}^{+\infty} \exp \left(-y^{2} / 2\right) \mathrm{d} y .
$$

It is recalled that a Gaussian random field is fully characterized by its translation-invariant covariance function, $\rho(\mathbf{r})=\mathbb{E}[Y(\mathbf{x}) Y(\mathbf{x}+\mathbf{r})]$. In turn, the two-point correlation function $S(\mathbf{r})$ of the resulting TGRF microstructure is related to $\rho(\mathbf{r})$ (Lantuejoul, 2002)

$$
S(\mathbf{r})=c-\frac{1}{2 \pi} \int_{\rho(\mathbf{r})}^{1} \exp \left(-\frac{\lambda^{2}}{1+t}\right) \frac{\mathrm{d} t}{\sqrt{1-t^{2}}} .
$$

In order to generate a TGRF microstructure with prescribed two point correlation function $S$ (r), we must first invert the above relation to find the covariance function $\rho(\mathbf{r})$ of the Gaussian random field $Y(\mathbf{x})$. Observing that the lag vector does not play any role in the above identity, we therefore seek the $S \mapsto \rho=R(S)$ mapping such that

$$
S=c-\frac{1}{2 \pi} \int_{R(S)}^{1} \exp \left(-\frac{\lambda^{2}}{1+t}\right) \frac{\mathrm{d} t}{\sqrt{1-t^{2}}} .
$$

This integral equation is equivalent to the following ordinary differential equation [where the unknown is the function $R(S)$ ]

$$
\frac{\mathrm{d} R}{\mathrm{~d} S}=2 \pi \sqrt{1-R^{2}} \exp \left(\frac{\lambda^{2}}{1+R}\right) \text { with } \quad R(c)=1 .
$$

For a given value of the threshold $\lambda$, the above ODE is solved numerically for $R(S)$. Then the covariance function of the Gaussian random field to be generated is given by $\rho(\mathbf{r})=R[S(\mathbf{r})]$. Generation of TGRF microstructures therefore proceeds as follows: 
a)

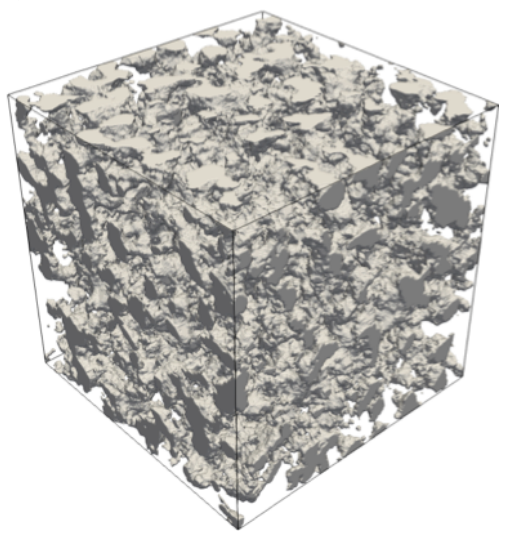

b)

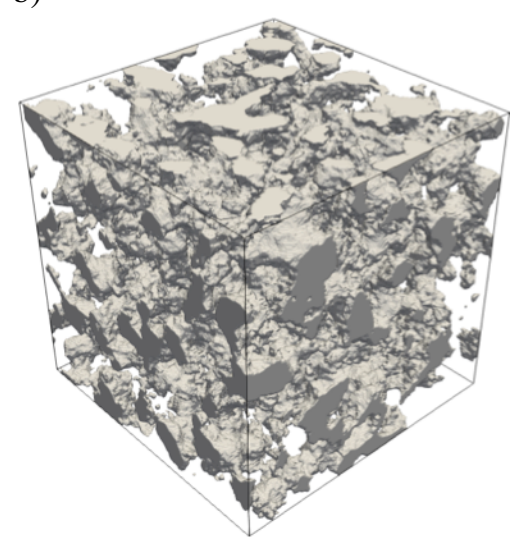

c)

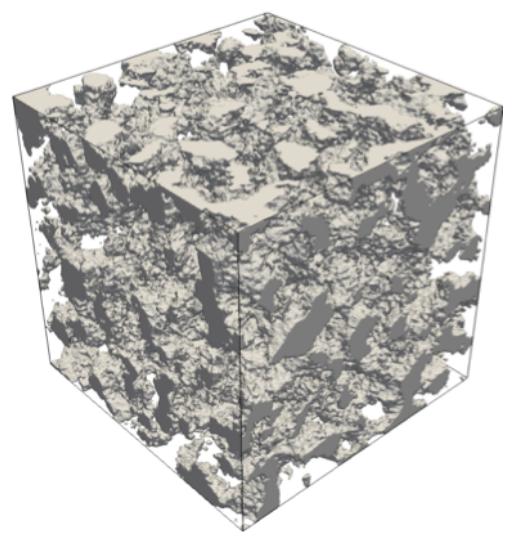

Figure 5: Examples of TGRF microstructures with porosity $c=25 \%$ reconstructed by using the two-point correlation functions of the RSA microstructures shown in Fig. 2 with void aspect ratio (a) $\omega=0.2$, (b) $\omega=0.4$ and (c) $\omega=1$.

1. Measure porosity $c$ and two-point correlation function $S(\mathbf{r})$ of the RSA microstructure to mimic.

2. Compute the threshold $\lambda$ and the mapping $S \mapsto R(S)$ from Eqs. (21) and (24).

3. Generate a Gaussian random field $Y$ with prescribed covariance $\rho(\mathbf{r})=R[S(\mathbf{r})]$.

4. Generate the porous microstructure $\chi_{\mathrm{p}}$ by thresholding $Y$ according to Eq. (19).

For point 1 , the target two-point correlation function $S(\mathbf{r})$ is obtained by averaging over 50 RSA realizations discretized over a $256^{3}$ cartesian grid. Various methods are available to achieve point 3 (see the review by Liu et al., 2019). In the present study, the random fields are discretized on a not-too-large cartesian grid (see Section 5.1). In turn, the spectral method based on the use of the fast Fourier transform (Shinozuka and Deodatis, 1991, 1996; Poirion and Soize, 1995) is found to provide a good compromise between ease of implementation and computational efficiency. Examples of generated TGRF microstructures are provided in Fig. 5.

\subsection{Computation of the effective elastic properties}

In this section, we discuss the computation of the effective linear elastic properties of the three types of porous microstructures presented in Secs. 3.1, 3.2 and 3.3. Without loss of generality, we consider the solid phase to be isotropic, linearly elastic described by a shear modulus $\mu_{\mathrm{s}}=0.492 \mathrm{GPa}$ and a Poisson's ratio $v_{\mathrm{s}}=0.42$. These values correspond to the experimentally measured properties of a 3d-printed Verowhite material (Zerhouni et al., 2019). It is recalled that the generated microstructures are periodic and thus periodic boundary conditions are applied to all full-field simulations presented below.

We use the numerical procedure initially introduced by Moulinec and Suquet $(1994,1998)$ to compute the effective elastic properties of the microstructures considered in this study. This method requires the microstructures to be discretized over a cartesian grid (all simulations presented here are conducted on $256^{3}$ grids). It relies on the reformulation of the classical homogenization problem (equilibrium under prescribed macroscopic strain $\bar{\varepsilon}$ and periodic boundary conditions) by the following equation

$$
\varepsilon+\Gamma_{0}\left[\left(\mathbb{C}-\mathbb{C}_{0}\right): \varepsilon\right]=\bar{\varepsilon}
$$

where $\mathbb{C}_{0}$ denotes the stiffness of an arbitrary reference material and $\boldsymbol{\Gamma}_{0}$ the associated fourth-order Green operator (see Appendix B). The $\boldsymbol{\Gamma}_{0}$ operator is a convolution operator and Eq. (25) is an integral equation. For the numerical solution of this equation, we use the variational approach of Brisard and Dormieux (2010, 2012), combining the discrete Green operator of Willot (2015) with a conjugate gradient linear solver. This approach relies on the equivalent formulation of Eq. (25)

$$
\left(\mathbb{C}-\mathbb{C}_{0}\right)^{-1}: \tau+\Gamma_{0}(\tau)=\bar{\varepsilon}, \quad \text { with } \quad \tau=\left(\mathbb{C}-\mathbb{C}_{0}\right): \varepsilon
$$


Here, $\tau$ denotes the stress-polarization, which is the principal unknown of the problem. For the reference material, we select a stiffness tensor $\mathbb{C}_{0}$ that is close to, but distinct from, that of the matrix. This avoids singularities due to the $\left(\mathbb{C}-\mathbb{C}_{0}\right)^{-1}$ factor.

For a given prescribed macroscopic strain $\bar{\varepsilon}$, the simulation returns the local stress-polarization $\tau$ at each grid-cell. The effective stiffness tensor $\widetilde{\mathbb{C}}$ is obtained from the volume average of Eq. (26)

$$
\langle\tau\rangle=\langle\mathbb{C}: \varepsilon\rangle-\left\langle\mathbb{C}_{0}: \varepsilon\right\rangle=\left(\widetilde{\mathbb{C}}-\mathbb{C}_{0}\right):\langle\boldsymbol{\varepsilon}\rangle=\left(\widetilde{\mathbb{C}}-\mathbb{C}_{0}\right): \bar{\varepsilon},
$$

where angle brackets $\langle\bullet\rangle$ refer to volume averages over the unit-cell. For each realization, the full, anisotropic, effective stiffness tensor $\mathbb{\mathbb { C }}$ is retrieved from simulations with six linearly independent values of the macroscopic strain $\overline{\boldsymbol{\varepsilon}}$. Note that for each set of microstructural parameters, 50 realizations were considered. These realizations were discretized over a $256^{3}$ cartesian grid. As a matter of fact, a smaller number of realizations may prove to be already sufficient (Anoukou et al., 2018) to ensure statistical convergence, especially in the context of linear elasticity.

It has already been noted in Section 5.1 that the microstructures considered here exhibit cubic symmetry that should evolve to isotropy for large unit-cells. In the present study, we generate microstructures that are large enough to be considered as representative volume elements (RVEs) (Ostoja-Starzewski, 2006) and the effective stiffness $\widetilde{\mathbf{C}}$ should exhibit almost perfect isotropy. To quantify this assertion, we introduce the following isotropic stiffness tensor

$$
\widetilde{\mathbb{C}}_{\text {iso }}=3 \tilde{\kappa} \mathbb{J}+2 \tilde{\mu} \mathbb{K}, \quad \text { with } \quad \tilde{\kappa}=\frac{1}{9} \widetilde{C}_{i i j j} \quad \text { and } \quad \tilde{\mu}=\frac{1}{10} \widetilde{C}_{i j i j}-\frac{3}{10} \tilde{\kappa},
$$

where $\mathbb{J}$ and $\mathbb{K}$ denote the spherical and deviatoric fourth-order projection tensors (see Appendix A). $\widetilde{\mathbb{C}}_{\text {iso }}$ is the isotropic tensor that is closest to $\widetilde{\mathbb{C}}$ in the sense of the Frobenius norm $\|\mathbb{A}\|_{\mathrm{F}}=\sqrt{A_{i j k l} A_{i j k l}}$. Then, the following dimensionless quantity

$$
\delta_{\text {iso }}=\frac{\left\|\widetilde{\mathbb{C}}-\widetilde{\mathbb{C}}_{\text {iso }}\right\|_{\mathrm{F}}}{\|\widetilde{\mathbb{C}}\|_{\mathrm{F}}}
$$

measures in a quantitative manner the deviation of the effective stiffness tensor $\widetilde{\mathbb{C}}$ from isotropy. For all microstructures considered in the present study, $\delta_{\text {iso }}$ was found to be lower than $3 \%$, while in most cases this deviation was even smaller than $1 \%$. Thus, the resulting deviation is deemed satisfactory, whereby detailed values of $\delta_{\text {iso }}$ are reported in Appendix C.

Note that the above-defined anisotropy index is more general than the Zener ratio, that applies to materials with cubic symmetry only. Also, it should be mentioned that $\delta_{\text {iso }}$ is not invariant by inversion: evaluating this index with the compliance tensor would likely have delivered a different value. Since the values of $\delta_{\text {iso }}$ effectively observed are rather small for all microstructures considered here, we did not find it necessary to introduce generalized euclidean distances that would preserve inversion (Morin et al., 2020).

In the following, we first investigate the effect of connectivity in the CRSA microstructures and subsequently, we compare all three microstructures in terms of their two-point correlation functions and the corresponding effective properties.

\section{A parametric study of the connectivity parameters}

In order to explore the effect of connectivity on the effective elastic properties, we carry out a fine control over the degree of connectivity in the microstructures under consideration. Such a parametric microstructural design is not possible with the TGRF microstructures (that are either percolated or not). This constitutes one of the main reasons for generating the CRSA microstructures, where channel size, described by $\eta$, and the average number of channels per pore, described by the coordination number $\zeta$, are introduced. In the present section, we show that even a relatively small volume fraction of connecting channels can have a significant effect on the effective elastic properties. It is important to mention at this point for clarity that introducing channels in the RSA microstructures leaves the twopoint correlations nearly unchanged (see for instance Fig. 10), as we will extensively discuss in the next Section 5.

The simulations in this section are performed for three porosities, $c=5 \%, 15 \%$ and $25 \%$. For all porosities, the observed trends were similar. The figures presented here therefore focus on the $c=25 \%$ microstructures, since larger porosities tend to emphasize the underlying microstructural effects and interactions between voids. To further 
a)

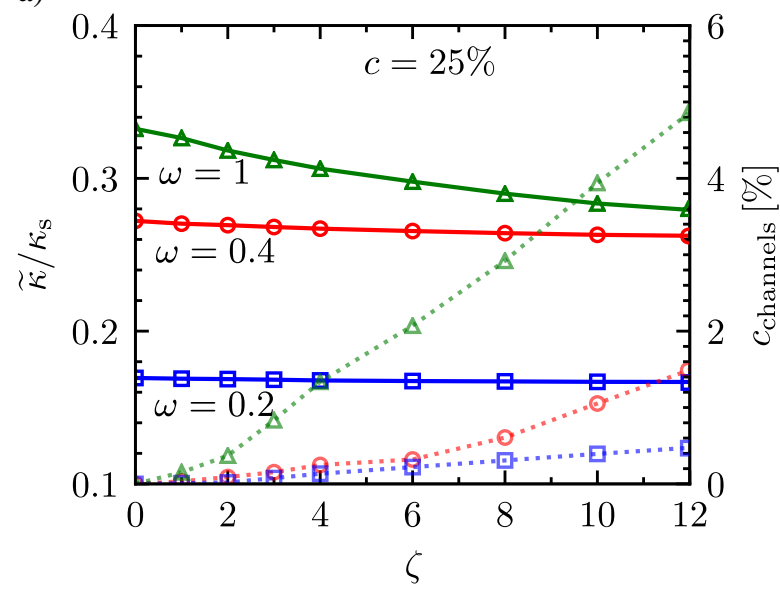

b)

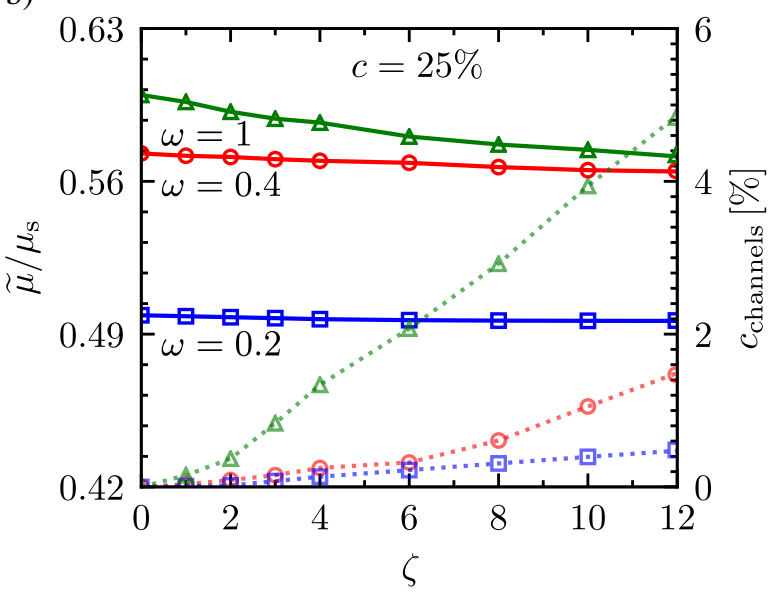

Figure 6: Effective moduli of the CRSA microstructures for fixed porosity $c=25 \%$ and reduced channel size $\eta=0.3$ and various values of the pore aspect ratio $\omega$ and coordination number $\zeta$. (a) effective bulk modulus. (b) Effective shear modulus. In both cases, the secondary right axis shows the volume fraction $c_{\text {channels }}$ of the cylindrical channels (dotted lines) as a function of the coordination number $\zeta$ for the same values of $c$ and $\eta$.

a)
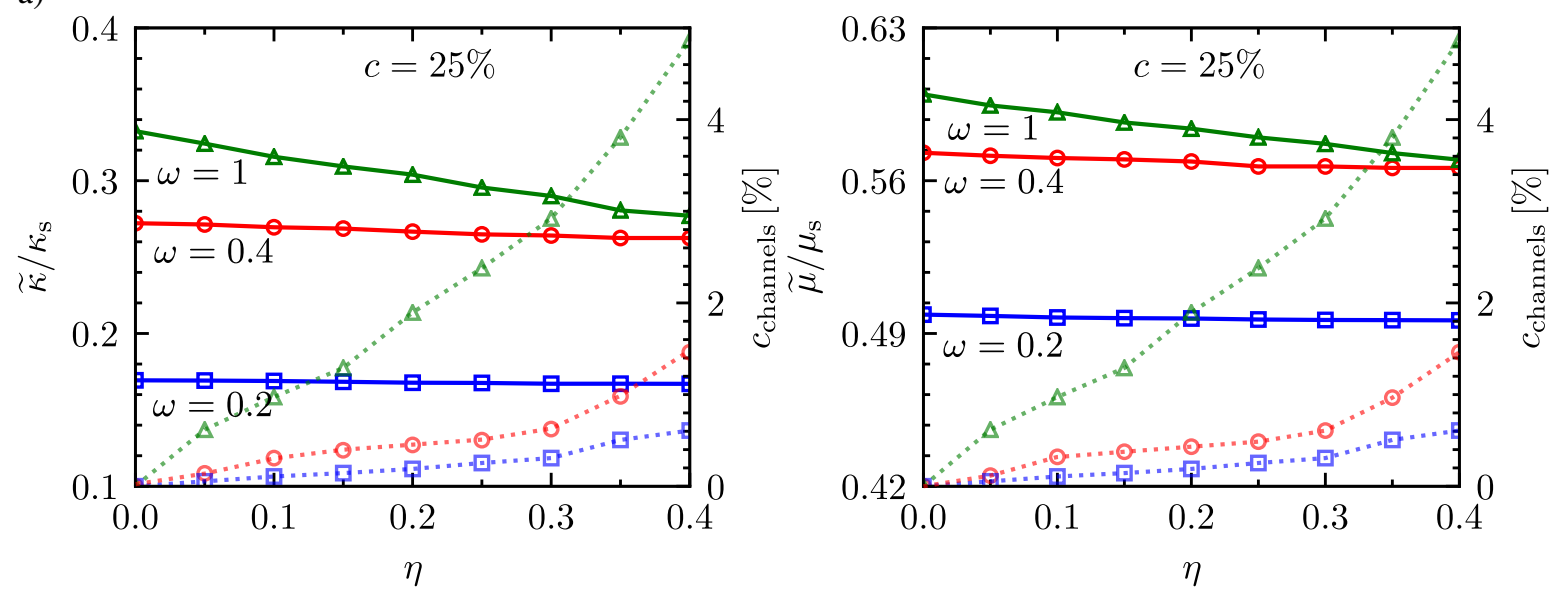

b)

Figure 7: Effective moduli of the CRSA microstructures for fixed porosity $c=25 \%$ and coordination number $\zeta=10$ and various values of the pore aspect ratio $\omega$ and reduced channel size $\eta$. (a) Effective bulk modulus. (b) Effective shear modulus. In both cases, the secondary right axis shows the volume fraction $c_{\text {channels }}$ of the cylindrical channels (dotted lines) as a function of the reduced channel size $\eta$ for the same values of $c$ and $\zeta$.

simplify the understanding of the obtained results, we introduce for this analysis an auxiliary post-measured parameter, the volume fraction $c_{\text {channels }}$ of channels, which depends on the two main ones $\eta$ and $\zeta$.

The influence of the coordination number $\zeta$ and channel size $\eta$ are analyzed in two series of simulations. In the first one, we fix the reduced channel size $\eta=0.3$ and vary the coordination number $\zeta$ from 0 up to the maximum value allowed by the available number of channel sites in the microstructure under consideration. Fig. 6 shows the resulting effective elastic moduli for $c=25 \%$ porosity and various values of the pore aspect ratio $\omega=0.2,0.4$ and 1 . In the second series of simulations, the coordination number is kept fixed $\zeta=10$, and the reduced channel size $\eta$ is varied from 0 to 0.4. The effective moduli are plotted in Fig. 7, again for $c=25 \%$ and $\omega=0.2,0.4$ and 1 .

Increasing $\zeta$ with $\eta$ fixed or increasing $\eta$ with $\zeta$ fixed leads in both cases to an increase in the volume fraction of channels $c_{\text {channels }}$ (see the secondary axis in Figs. 6 and 7). Note, however, that the total porosity remains fixed. In both cases, this induces a decrease of the effective elastic moduli. The effect on the effective bulk modulus is very significant, especially for spherical voids corresponding to $\omega=1$. The effect of connectivity becomes gradually less significant with decreasing $\omega$. As an example, a $15 \%$ reduction is observed for $\widetilde{\kappa}$ and $\omega=1$. Instead, for 
$\omega=0.2$, the effective bulk modulus remains fairly unchanged with connectivity. It should be noted, however, that as the voids become more flat, the volume fraction of channels becomes significantly smaller. The reason for such a behavior is related to the fact that as the voids become flatter $(\omega \ll 1)$, the distance between two neighboring voids in some direction becomes much shorter than that in spherical voids. As a consequence, the connection is achieved with a much smaller channel length, leading to smaller channel porosity in total. One could introduce a different strategy to connect the voids introducing connectivity at larger distances. Such an approach, albeit easily envisaged, is not considered in the present work since the goal is to have a tractable minimum set of microstructural parameters. Interestingly, the effect of connectivity on the effective shear modulus is much more limited, leading to a maximum $5 \%$ reduction in the case of spherical voids.

a)

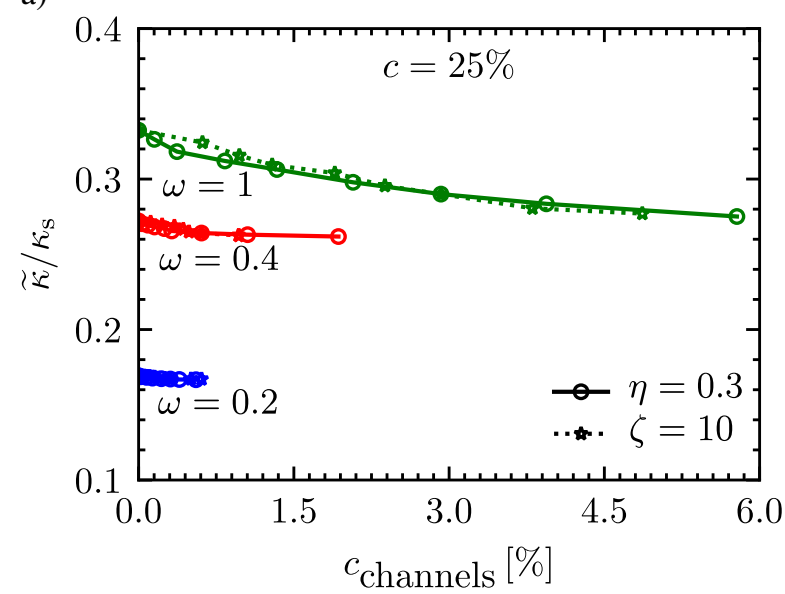

b)

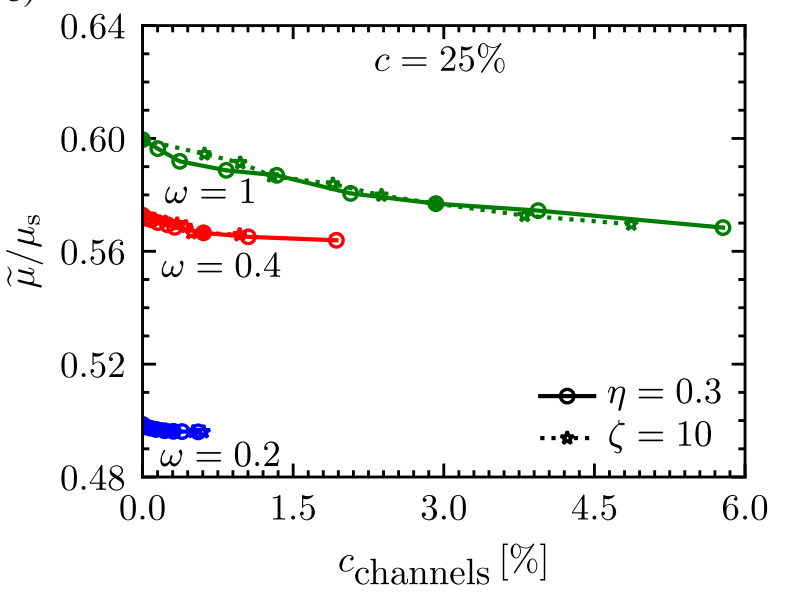

Figure 8: Effective moduli of the CRSA microstructures for fixed porosity $c=25 \%$ and various values of the pore aspect ratio $\omega$, coordination number $\zeta$ and reduced channel size $\eta$. Solid lines: $\eta=0.3, \zeta$ varies as in Fig. 6; dotted lines: $\zeta=10$ and $\eta$ varies as in Fig. 7 . (a) Effective bulk modulus. (b) Effective shear modulus.

In order to reveal more clearly the relative weight of the coordination number $\zeta$ and the reduced channel size $\eta$, we cross-plot in Fig. 8 the effective elastic moduli obtained in both series of simulations, as a function of the channel volume fraction, $c_{\text {channels. }}$ It is observed that the curves for $\eta$ fixed and $\zeta$ fixed overlap with an excellent precision. This seems to indicate that $c_{\text {channels }}$ is the first-order parameter in this elasticity problem. This conclusion is somewhat different than that by Nirmalraj et al. (2012), where it was shown that $\zeta$ is the dominant parameter in the context of connected nano-wires. Nevertheless, the latter microstructure is substantially different than the one considered here, where the main ingredient is the original ellipsoidal voids, which are subsequently connected by cylindrical channels. This observation suggests strongly that the effect of connectivity is highly sensitive to the particular details of each microstructure.

\section{The effect of the two-point correlations on the effective elastic properties}

In this section, we analyze the effect of the two-point correlation functions on the effective elastic properties using the three microstructures discussed in Secs. 3.1, 3.2 and 3.3. We carry out first a statistical analysis in Section 5.1 and subsequently, we evaluate in Section 5.2 the corresponding effective bulk and shear moduli resulting from those microstructures.

\subsection{Statistical analysis of the generated microstructures}

All microstructures generated in Secs. 3.1, 3.2 and 3.3 are discretized over a $256^{3}$ cartesian grid prior to any further analysis. This leads to a cell-size $h \simeq a_{\min } / 6(c=5 \%), h \simeq a_{\min } / 8(c=15 \%)$ and $h \simeq a_{\min } / 10(c=25 \%)$, where $a_{\min }$ denotes the minimum radius over all spheroidal pores

$$
a_{\min }=\min \left\{a_{1}^{(r)}, a_{2}^{(r)}, a_{3}^{(r)}, 1 \leq r \leq N_{\text {phases }}\right\} .
$$


The two-point correlation functions are then computed by means of discrete Fourier transforms (using a fast Fourier transform implementation)

$$
S^{h}=N_{\text {cells }}^{-1} \operatorname{DFT}^{-1}\left[\left|\operatorname{DFT}\left(\chi^{h}\right)\right|^{2}\right]
$$

where $N_{\text {cells }}$ denotes the total number of grid cells, $\chi^{h}$ denotes the indicator function of the pore space, discretized over the cartesian grid, and $S^{h}$ the resulting discretized two-point correlation function. An empirical mean is then computed over 50 realizations for each set of microstructural parameters.

For finite-size unit-cells, only cubic symmetry is to be expected for $S^{h}$. However, for sufficiently large unit-cells $(L \rightarrow+\infty)$, the computed two-point correlation function $\mathrm{S}(\mathrm{r})$ should be nearly isotropic for small magnitudes of the lag-vector $\mathbf{r}$. This is verified empirically by comparing 1D sections of $S^{h}$ along the three axes of the box; Figs. 9 and 10 actually display the following mean

$$
S^{h}(r)=\frac{1}{3}\left[S^{h}(r, 0,0)+S^{h}(0, r, 0)+S^{h}(0,0, r)\right] .
$$

Note that, owing to the periodicity of the generated microstructures, the correlation functions are symmetric about $r=L / 2: S^{h}(L-r)=S^{h}(r)$. Therefore, in Figs. 9 and 10, $S^{h}(r)$ is plotted only for $0 \leq r \leq L / 2$. The correlation length $\ell_{\mathrm{c}}$ introduced in Table 2 is defined as follows

$$
\ell_{\mathrm{c}}=\int_{0}^{L / 2} S^{h}(r) \mathrm{d} r .
$$

For the sake of simplicity, the $h$ superscript will be omitted in the remainder of this paper, keeping in mind that all fields are discretized over a $256^{3}$ cartesian grid.

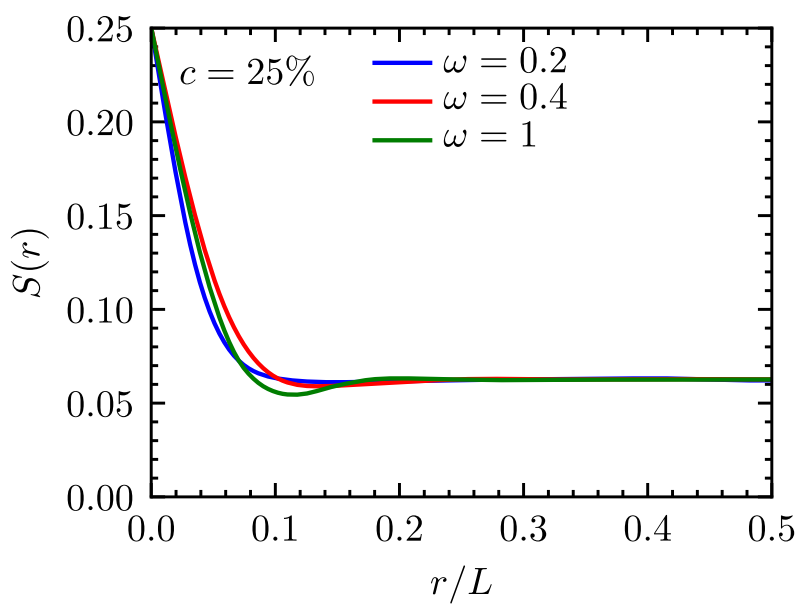

Figure 9: Two-point correlation functions of the RSA microstructures for porosity $c=25 \%$ and pore aspect ratios $\omega=0.2,0.4$ and 1.

The two-point correlation function of the RSA microstructures is displayed in Fig. 9, for a total porosity $c=25 \%$ and various values of the aspect ratio $\omega$. It is observed that for assemblies of spheres, a strong correlation occurs at $r / L \simeq 0.1$. For anisotropic pores $(\omega<1)$, polydispersity (in orientations) tends to flatten the $S(r)$ curves.

\begin{tabular}{c|ccc}
\hline$\omega$ & $\ell_{\mathrm{c}} S_{\mathrm{v}}(\mathrm{RSA})$ & $\ell_{\mathrm{c}} S_{\mathrm{v}}(\mathrm{CRSA})$ & $\ell_{\mathrm{c}} S_{\mathrm{v}}(\mathrm{TGRF})$ \\
\hline 0.2 & 0.582 & 0.591 & 0.582 \\
0.4 & 0.449 & 0.478 & 0.448 \\
1.0 & 0.476 & 0.532 & 0.478 \\
\hline
\end{tabular}

Table 2: Comparison of the specific surface area $S_{\mathrm{v}}$ for a porosity $c=15 \%$ and three values of the aspect ratio $\omega$. Values of $S_{\mathrm{v}}$ are reported in dimensionless form through the product $\ell_{\mathrm{c}} S_{\mathrm{v}}$, where $\ell_{\mathrm{c}}$ denotes the correlation length defined through equation (33).

Fig. 10 shows the two-point correlation functions for all three types of microstructures, $c=25 \%$ and $\omega=0.2,1$. As expected, although visually different if one observes Figs. 2 and 5, the RSA and GRF microstructures exhibit by 
a)

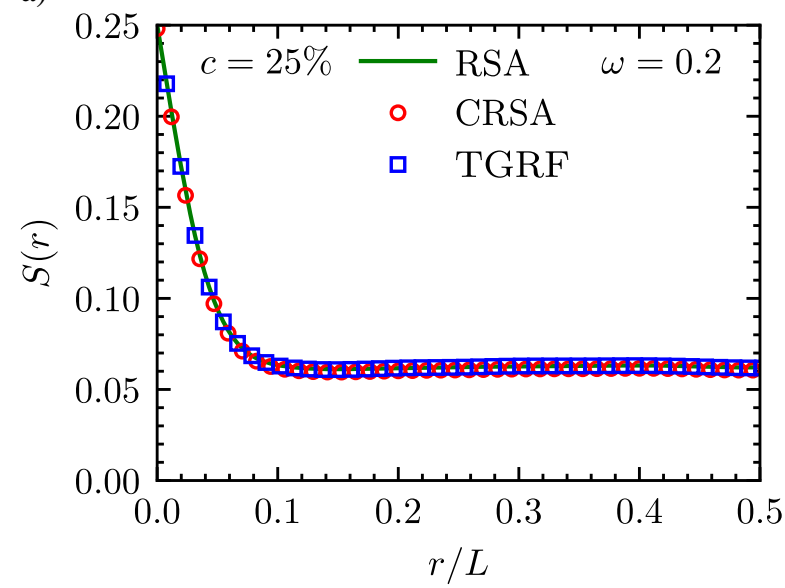

b)

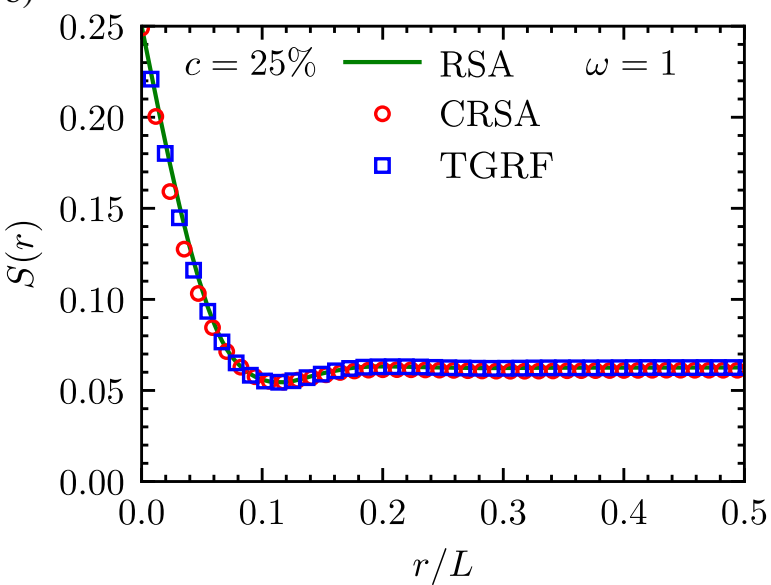

Figure 10: Comparison of the two-point correlations for the three types of microstructures (RSA, CRSA and TGRF), with $c=25 \%$ and various values of $\omega$. (a) $\omega=0.2$. (b) $\omega=1$. For the CRSA microstructures, $\eta=0.3$ and $\zeta=6$.

construction almost identical two-point correlation functions. More surprising though is the fact that the introduction of channels in the CRSA microstructures did not alter the two-point correlations, at least up to an extremely weak percentage. This is quantitatively confirmed by the evaluation of the specific surfaces $S_{\mathrm{v}}$ which are fairly close, as shown in Table 10.

\subsection{Effective elastic properties of the generated microstructures}

In the previous Section 5.1, we have shown that the three generated families of microstructures, RSA, TGRF and CRSA, exhibit almost the same second-order statistics, even though they are morphologically fairly different. We now confront these purely morphological results to the corresponding mechanical elasticity results.

Fig. 11 shows the effective bulk and shear moduli $\tilde{\kappa}$ and $\tilde{\mu}$ for the RSA, CRSA and TGRF microstructures as a function of the porosity $c$, for various values of the void aspect ratio $\omega=1,0.4,0.2$. The effective values are normalized by the elastic moduli of the solid phase, $\kappa_{\mathrm{s}}$ and $\mu_{\mathrm{s}}$ and for the CRSA microstructures, $\eta=0.25$ and $\zeta=4$.

We observe that the case of spherical voids $\omega=1$ (Fig. 11a,b) is the case for which the three analyzed microstructures deliver estimates that differ most. The differences are more pronounced for the bulk modulus than for the shear modulus. In this case, the TGRF microstructure leads to the lowest values and the RSA to the stiffest ones. In fact, in several studies (see for instance Anoukou et al., 2018), it was shown that the RSA microstructure is very close to the Hashin-Shtrikman bounds (Hashin and Shtrikman, 1963) for isotropic porous materials. Decreasing the void aspect ratio to $\omega=0.4$ (Fig. 11c,d) leads to a particular case where the bulk modulus predicted by all microstructures is fairly close for all porosities. This observation, however, does not carry on entirely to the corresponding shear modulus. Finally, as we further decrease the aspect ratio to $\omega=0.2$ (Fig. 11e,f), we observe that the RSA and CRSA curves almost overlap for both the bulk and shear moduli, indicating that connectivity has a minor effect in this case. In turn, the TGRF microstructure is much stiffer than the RSA and CRSA microstructures in this case. Interestingly, the maximum difference between the RSA (resp. CRSA) and the TGRF microstructures is observed for porosities in the order of $\sim 10 \%$.

In order to have a more complete perspective of those results, Fig. 12 presents a cross-plot of the same normalized effective bulk and shear moduli as a function of $\omega$ and for three selected porosities $c=5,15$ and $25 \%$. For clarity, we also include the partial channel porosity $c_{\text {channels }}$ for the CRSA as a secondary axis in all plots. We also note that for the CRSA microstructures, $\eta=0.25$ and $\zeta=4$.

We immediately observe that the effective bulk modulus is more sensitive to the aspect ratio $\omega$ than the shear modulus. In addition, for aspect ratios $\omega<0.4$, the introduction of connectivity moves the CRSA estimates towards the TGRF ones, making the overall response of the CRSA material softer than the RSA one. This effect is rather strong for the bulk modulus, where for $\omega=1$ the difference between the RSA and CRSA microstructures is more than 
(a)

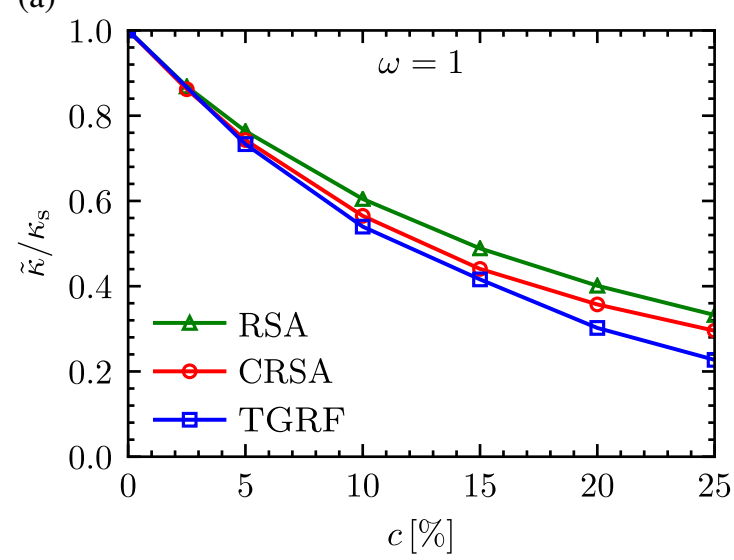

(c)

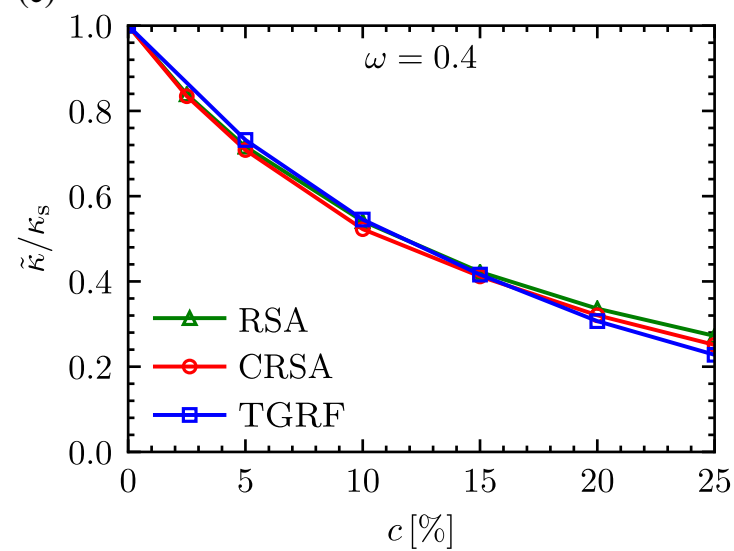

(e)

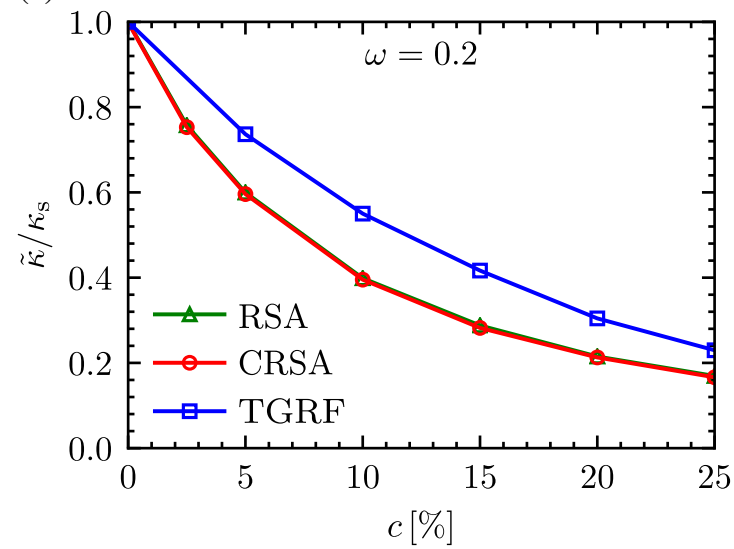

(b)

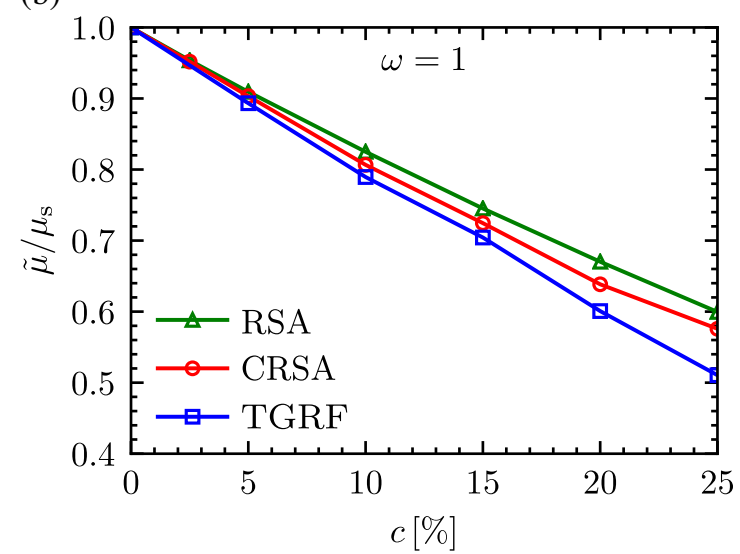

(d)

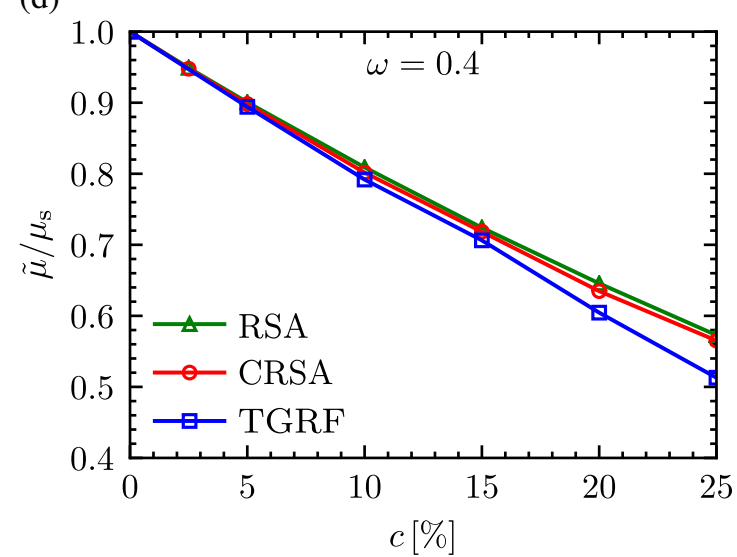

(f)

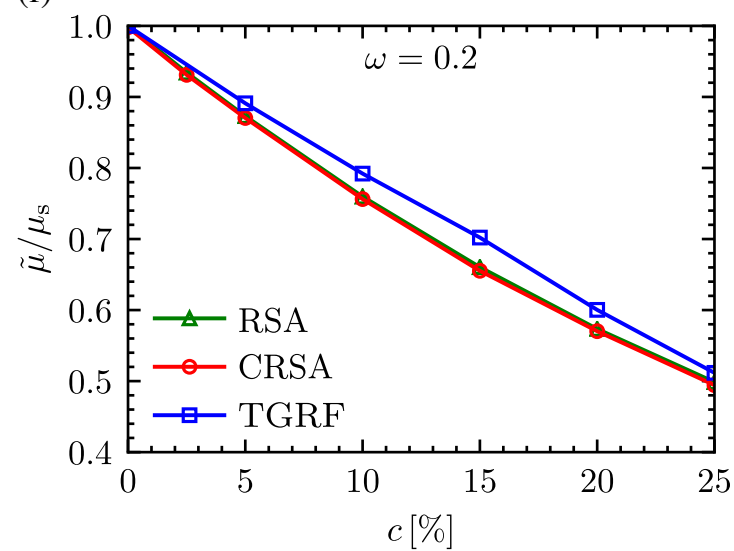

Figure 11: Effective normalized bulk $\tilde{\kappa} / \kappa_{\mathrm{S}}$ and shear $\tilde{\mu} / \mu_{\mathrm{s}}$ moduli of the RSA, TGRF and CRSA microstructures as a function of the porosity $c$, for a pore aspect ratio $(\mathrm{a}, \mathrm{b}) \omega=1,(\mathrm{c}, \mathrm{d}) \omega=0.4$ and $(\mathrm{e}, \mathrm{f}) \omega=0.2$. For the CRSA microstructures, $\eta=0.25$ and $\zeta=4$.

$13 \%$ for $c=15 \%$ (Fig. 12c). In this case, $c_{\text {channels }}$ is also the largest. Similar trends are obtained also for the shear modulus, but the effect of connectivity is somewhat less pronounced. In turn, the effect of the aspect ratio becomes extremely critical for $\omega<0.3$. 
(a)

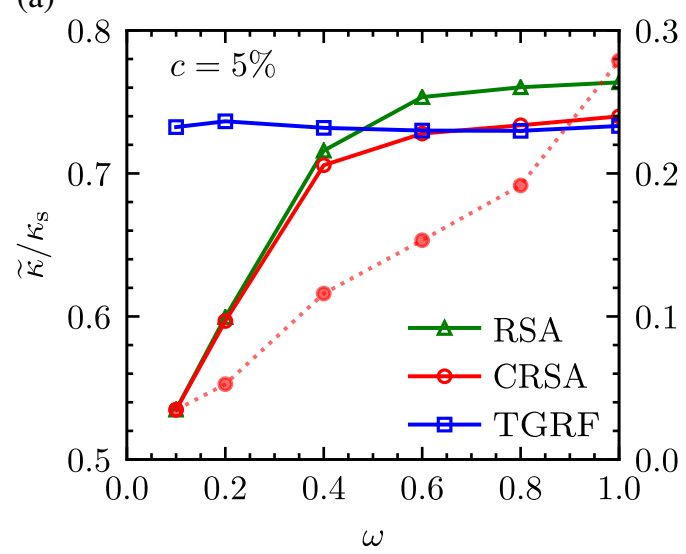

(c)

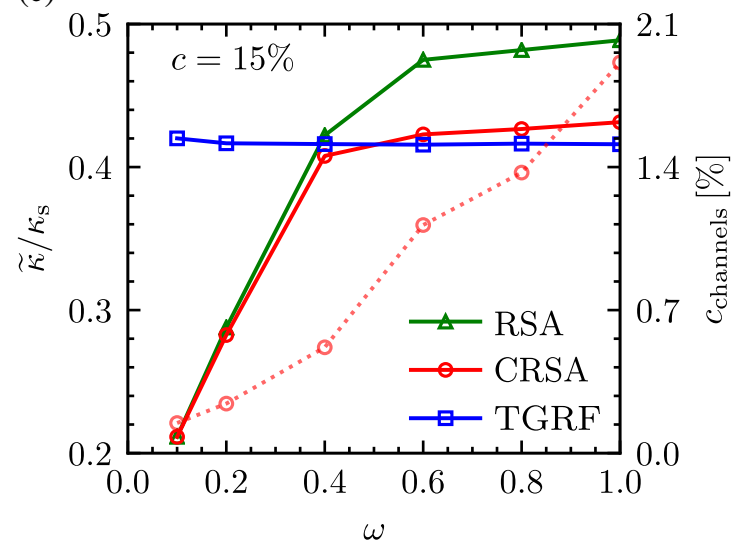

(e)

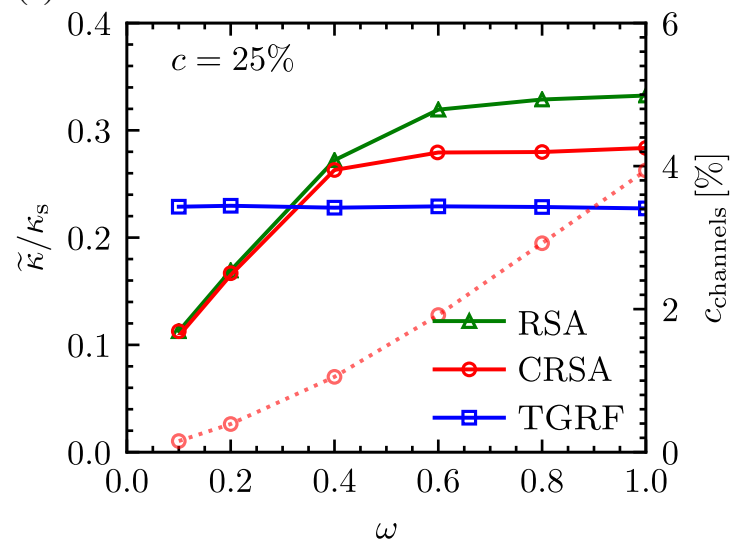

(b)

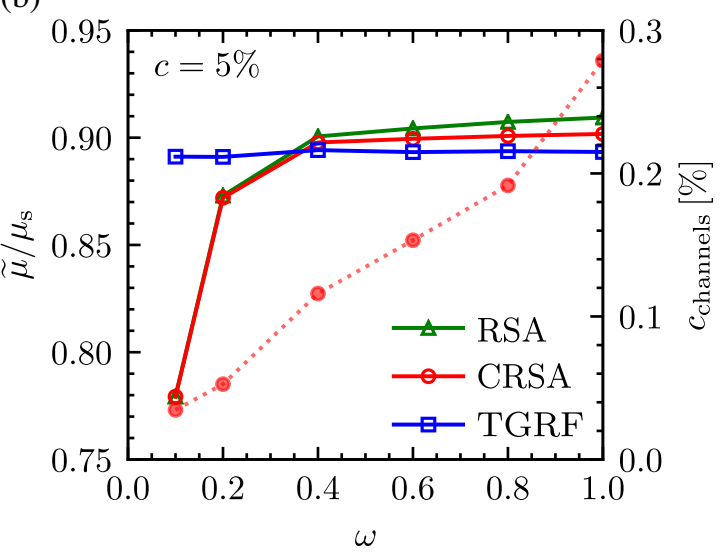

(d)

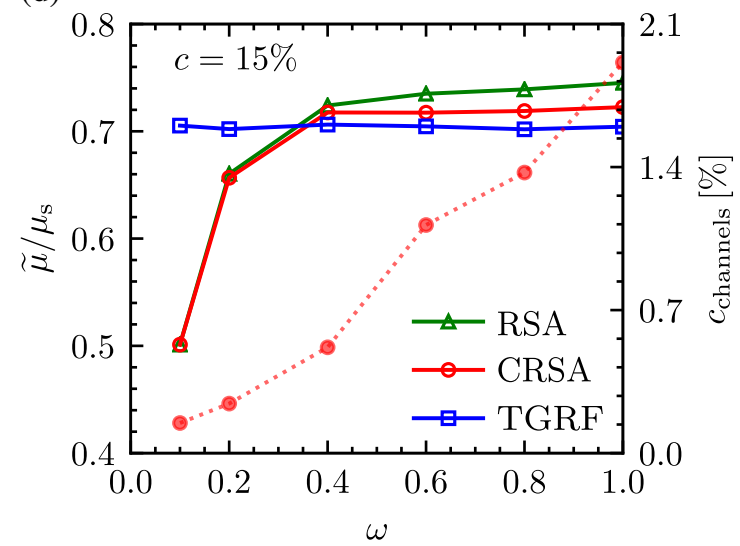

(f)

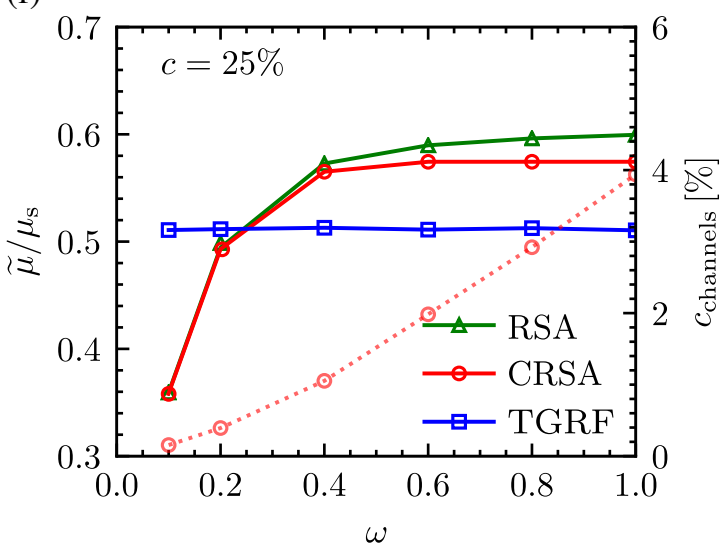

Figure 12: Effective normalized bulk $\tilde{\kappa} / \kappa_{\mathrm{S}}$ and shear $\tilde{\mu} / \mu_{\mathrm{s}}$ moduli of the RSA, TGRF and CRSA microstructures as a function of the void aspect ratio $\omega$ for various porosities $(\mathrm{a}, \mathrm{b}) c=5 \%,(\mathrm{c}, \mathrm{d}) c=15 \%$ and $(\mathrm{e}, \mathrm{f}) c=25 \%$. For the CRSA microstructures, $\eta=0.25$ and $\zeta=4$. The secondary right axis shows the partial channel porosity $c_{\text {channels }}$ for the CRSA.

\subsection{Discussion on the relevance of the two-point correlations and connectivity}

In view of these results, one may clearly state that connectivity indeed plays a significant role on the effective elastic properties, particularly for the effective bulk modulus, and that differences in connectivity could explain why the RSA and TGRF microstructures exhibit so different effective elastic properties while sharing the same two-point correlations. In particular, the RSA microstructures are fully disconnected, whereas the TGRF ones have some degree 
of connectivity (depending on the volume fraction) and can even be percolated (Tomita and Murakami, 1988; Øren and Bakke, 2002; Roubin and Colliat, 2016).

It is known that the effective conductivity and bulk modulus have similar behaviors (one could compare for example the three-point bounds on these two quantities in Torquato and Lado (1986)). The decrease of the effective bulk modulus observed here with an increasing volume fraction of connecting channels is therefore consistent with previous studies of the effective conductivity of two-phase materials, where connectivity was shown to play a central role (Stenzel et al., 2016; Neumann et al., 2020). It is also consistent with recent observations by Cai et al. (2019) regarding the effective elastic properties. The present study further shows that the effective shear modulus is less affected by connectivity than the bulk modulus.

We also conclude that, from the point of view of solid mechanics, the two-point correlations are highly insufficient descriptors. Although not shown here in order not to scramble the main message of this study, we recall that the general expression of the Hashin-Shtrikman bounds on the effective properties include the two-point correlation functions (Willis, 1977). However, for isotropic microstructures, these functions vanish from the resulting bound. Therefore, the bounds on the effective properties coincide for all isotropic microstructures. While this universality may be regarded as a strength of the Hashin-Shtrikman bounds, in the sense of being able to bound an immense set of isotropic porous materials, from the perspective of this paper, it shows, in turn, that the two-point correlation functions are not relevant descriptors of the microstructure. By contrast, one could have used the more elaborate Hashin-Shtrikman-Willis estimates (Willis, 1981) (with their projection in the isotropic space as in Gatt et al., 2005), which allow to describe directly the void aspect ratio and their distributions (Ponte Castañeda and Willis, 1995). Nevertheless, such a model would be only capable to deliver estimates for the RSA microstructures, as already shown in Anoukou et al. (2018). It would be inappropriate for the CRSA and TGRF microstructures.

From a different regard, Torquato (1997) expressed the effective elastic properties of two-phase materials as a series expansion in powers of the so-called "elastic polarizabilities" (a normalized difference of the elastic moduli of the two phases). The most notable result of this formal study is that the term of order $k$ involves the correlation functions up to order $k$. These expansions were specialized to isotropic materials by Torquato (1998), where it was shown that the term of second order (which should therefore involve the two-point correlations only) vanished in that case. In other words, from the perspective of the effective elastic properties, the two-point correlation functions alone do not carry more information than the volume fractions, which is consistent with what is observed numerically here and confirms that the two-point correlation functions are not relevant descriptors of the microstructure.

\section{Concluding remarks}

In the present study, we propose a quantitative investigation of the influence of the microstructure on the effective elastic properties of porous materials. In particular, we focus the study on the question of whether the two-point correlations in a given microstructure are sufficient to describe accurately its effective elastic properties. In order to address this question unambiguously in the context of linear elasticity, we use three paradigms of well-defined microstructures that share almost identical two-point statistics but are morphologically different. The first microstructure comprises non-overlapping polydisperse spherical and ellipsoidal voids oriented randomly. Its construction is achieved by use of the random sequential adsorption (RSA) algorithm. The second microstructure, termed connected random sequential adsorption (CRSA) and introduced for the first time in this work, derives directly from the RSA by introducing cylindrical with circular cross-section porous channels that connect the existing spherical or ellipsoidal voids. The third microstructure, based on a thresholded Gaussian random field (TGRF) process, is constructed on purpose by direct use of the numerical two-point correlations of the RSA microstructure. As a result, the RSA and TGRF have almost identical one- and two-point correlations. Perhaps more interestingly, we also find that the introduction of connectivity to obtain the CRSA microstructure affects very weakly the two-point correlations. These paradigms-which are extensively used to model porous materials spanning geomaterials, ceramics, wood and so on-allow to have three rather different microstructures in terms of connectivity and more generally local geometric features but with the same two-point statistics.

Using next FFT numerical simulations, we compute and compare the effective bulk and shear moduli in those three cases. In this study, we show by direct comparison that the two-point correlations alone are insufficient to describe quantitatively the effective elastic properties of porous materials in general. The three microstructures used in this study are found to exhibit significantly different effective elastic properties, and especially different bulk moduli, even 
if their two-point statistics are almost identical. More precisely, we observe that connectivity in the CRSA materials tends to decrease the effective properties when compared with the original RSA microstructure. In particular, we find that even a relatively small number of connected regions in the closest neighborhood of a given void is sufficient to lead to a significant drop of the effective elastic properties (see also recent work by Cai et al., 2019, along these lines). Moreover, we find that in the present CRSA procedure the dominant parameter is the volume fraction of the connecting channels. In turn, the TGRF microstructures are found to be softer than both the RSA and the CRSA for void aspect ratios between 0.4 (oblate) and 1 (spherical voids). Instead, as the void aspect ratio decreases to values less than 0.3 , the RSA and CRSA become increasingly softer reaching very low values for the effective bulk and shear moduli, much lower than the corresponding TGRF ones.

The versatile and highly non-trivial effect of the local microstructural features on the effective elastic properties reveals simply that a wide number of extensively used analytical micromechanical models, that are based on twopoint correlations or an ideal inclusion of ellipsoidal shape (e.g., self-consistent estimates, Hashin-Shtrikman bounds, Mori-Tanaka and many other) have a very specific range of validity and should be used with extreme caution, especially when comparison is attempted with experiments, where the material microstructure is extremely complex. In particular, in a large number of geological and metallic materials, the voids exhibit irregular shapes (see for instance the TGRF microstructures shown above) and more often than not are connected in several regions (e.g. due to fracturing or coalescence). While identifying an ideal ellipsoidal shape that fits the elastic response may prove useful in certain circumstances, such a practice can lead to substantial errors when permeability or nonlinear effects (such as plasticity, nonlinear elasticity etc) need to be modeled. This in fact may explain partially the very large span of models that have been proposed in the context of analytical linear and nonlinear homogenization, as well as their difficulty to model actual materials at small and finite strains. From a different point of view, the present results indicate that slight perturbations in the initial conditions during an optimization process may lead to microstructures that deliver substantially different effective properties. Finally, we note that while the present work focuses on isotropic porous materials, we expect that similar conclusions may be drawn for other types of large contrast microstructures, such as anisotropic ones, particle reinforced composites and multi-phase materials. Nevertheless, a complete study along these lines needs to be carried out to validate without doubt such a proposition.

\section{Acknowledgments}

The authors acknowledge support from TOTAL S.A. K.D. also acknowledges support of the European Research Council (ERC) under the European Union's Horizon 2020 research and innovation program (grant agreement no. 636903). The computational part of this work was also supported by the ANR under contract number ANR-10EQPX-37.

\section{A. The fourth-order isotropic projection tensors}

Let $\mathbf{I}$ denote the second-order identity tensor and $\mathbb{I}$ the fourth-order identity tensor over the space of symmetric, second-order tensors

$$
\mathbf{I}=\delta_{i j} \mathbf{e}_{i} \otimes \mathbf{e}_{j} \quad \text { and } \quad \mathbb{I}=\frac{1}{2}\left(\delta_{i k} \delta_{j l}+\delta_{i l} \delta_{j k}\right) \mathbf{e}_{i} \otimes \mathbf{e}_{j} \otimes \mathbf{e}_{k} \otimes \mathbf{e}_{l} .
$$

The spherical and deviatoric fourth-order projection tensors $\mathbb{J}$ and $\mathbb{K}$ are defined as follows

$$
\mathbb{J}=\frac{1}{3} \mathbf{I} \otimes \mathbf{I}, \quad \mathbf{K}=\mathbb{I}-\mathbb{J}, \quad \text { with } \quad \mathbb{J}: \mathbb{J}=\mathbb{J}, \quad \mathbb{K}: \mathbb{K}=\mathbb{K}, \quad \mathbb{J}: \mathbb{K}=\mathbb{K}: \mathbb{J}=\mathbb{O} .
$$

It is known that any fourth-order, isotropic tensor with minor symmetries is a linear combination of these two projectors (Walpole, 1984).

\section{B. The fourth-order Gamma operator}

For periodic boundary conditions, the classical homogenization problem reads

$$
\operatorname{div}\left[\mathbb{C}:\left(\overline{\boldsymbol{\varepsilon}}+\operatorname{sym} \operatorname{grad} \mathbf{u}^{\text {per }}\right)\right]=\mathbf{0},
$$


where $\mathbf{u}^{\text {per }}$ is a periodic displacement field, to be determined. In the above problem, the stiffness $\mathbb{C}$ of the heterogeneous material is a field, that depends on the observation point. When the material is homogeneous with stiffness $\mathbb{C}_{0}$, the above problem is trivial $\left(\mathbf{u}^{\text {per }}=\mathbf{0}\right)$. A closely related problem can be constructed in that case with the introduction of a so-called stress-polarization field $\tau$

$$
\operatorname{div}\left[\mathbb{C}_{0}:\left(\operatorname{sym} \operatorname{grad} \mathbf{u}^{\text {per }}\right)+\tau\right]=\mathbf{0} .
$$

Note that in the above problem, the material is homogeneous and the macroscopic strain is null. Owing to linearity, the solution to Problem (B.2) depends linearly on the parameter $\tau$ (which is a tensor field). In particular, the total strain sym grad $\mathbf{u}^{\text {per }}$ (also a field) depends linearly on $\tau$. Following Korringa (1973), Zeller and Dederichs (1973) and Kröner (1974), the Gamma operator is defined as the operator that maps linearly the stress-polarization onto the opposite of the induced strain tensor, i.e.,

$$
\operatorname{sym} \operatorname{grad} \mathbf{u}^{\text {per }}=-\boldsymbol{\Gamma}_{0}(\tau) .
$$

It is again emphasized that both $\tau$ and $\boldsymbol{\Gamma}_{0}(\tau)$ are (second-order, symmetric) tensor fields. The Gamma operator has a number of properties that can be found in e.g. Michel et al. (2001).

\section{On the mechanical isotropy of the generated microstructures}

In this appendix, we report the values of $\delta_{\text {iso }}$ for the microstructures considered in the present article. It is recalled that $\delta_{\text {iso }}$ is defined in Section 3.4 and measures the deviation of the effective stiffness tensor from its isotropic projection. In other words, smaller values of $\delta_{\text {iso }}$ refer to "more elastically isotropic" microstructures. Note that the degree of linear elastic isotropy that is measured here is less stringent than the rotational invariance of the correlation functions, which defines a statistically isotropic microstructure.

The values of $\delta_{\text {iso }}$ are reported in table C. 3 for the RSA microstructures, table C. 4 for the CRSA microstructures and table C.5 for the TGRF microstructures. We observe that in all cases, we obtain sufficiently isotropic microstructures, with the maximum deviation obtained for the TGRF unit cells and porosity $c=25 \%$.

\begin{tabular}{c|ccc}
\hline RSA & $\omega=0.2$ & $\omega=0.4$ & $\omega=1$ \\
\hline$c=5 \%$ & $0.21 \%$ & $0.12 \%$ & $0.04 \%$ \\
$c=15 \%$ & $0.66 \%$ & $0.60 \%$ & $0.17 \%$ \\
$c=25 \%$ & $2.06 \%$ & $1.76 \%$ & $0.32 \%$ \\
\hline
\end{tabular}

Table C.3: Values of $\delta_{\text {iso }}$ for the RSA microstructures, for various values of the porosity $c$ and pore aspect ratio $\omega$.

\begin{tabular}{c|ccc}
\hline CRSA & $\omega=0.2$ & $\omega=0.4$ & $\omega=1$ \\
\hline$c=5 \%$ & $0.88 \%$ & $0.40 \%$ & $0.12 \%$ \\
$c=15 \%$ & $1.74 \%$ & $1.11 \%$ & $0.43 \%$ \\
$c=25 \%$ & $2.36 \%$ & $1.28 \%$ & $0.55 \%$ \\
\hline
\end{tabular}

Table C.4: Values of $\delta_{\text {iso }}$ for the CRSA microstructures, for various values of the porosity $c$ and pore aspect ratio $\omega$. For all microstructures, $\eta=0.25$ and $\zeta=4$.

\begin{tabular}{c|ccc}
\hline TGRF & $\omega=0.2$ & $\omega=0.4$ & $\omega=1$ \\
\hline$c=5 \%$ & $0.17 \%$ & $0.12 \%$ & $0.04 \%$ \\
$c=15 \%$ & $0.71 \%$ & $0.60 \%$ & $0.17 \%$ \\
$c=25 \%$ & $2.65 \%$ & $1.76 \%$ & $0.35 \%$ \\
\hline
\end{tabular}

Table C.5: Values of $\delta_{\text {iso }}$ for the TGRF microstructures, for various values of the porosity $c$ and pore aspect ratio $\omega$ 


\section{References}

Ali, M.N., Rehman, I.U., 2011. An auxetic structure configured as oesophageal stent with potential to be used for palliative treatment of oesophageal cancer; development and in vitro mechanical analysis. Journal of materials science. Materials in medicine 22, $2573-2581$.

Altendorf, H., Jeulin, D., Willot, F., 2014. Influence of the fiber geometry on the macroscopic elastic and thermal properties. International Journal of Solids and Structures 51, $3807-3822$.

Anoukou, K., Brenner, R., Hong, F., Pellerin, M., Danas, K., 2018. Random distribution of polydisperse ellipsoidal inclusions and homogenization estimates for porous elastic materials. Computers \& Structures 210, 87-101.

Ashby, M., 2013. Designing architectured materials. Scripta Materialia 68, 4-7. Architectured Materials.

Baniassadi, M., Addiego, F., Laachachi, A., Ahzi, S., Garmestani, H., Hassouna, F., Makradi, A., Toniazzo, V., Ruch, D., 2011. Using SAXS approach to estimate thermal conductivity of polystyrene/zirconia nanocomposite by exploiting strong contrast technique. Acta Materialia 59, 2742-2748.

Benveniste, Y., 1987. A new approach to the application of Mori-Tanaka's theory in composite materials. Mechanics of Materials 6, $147-157$.

Berryman, J.G., 1985. Measurement of spatial correlation functions using image processing techniques. Journal of Applied Physics 57, $2374-2384$.

Berryman, J.G., Pride, S.R., Wang, H.F., 2002. A differential scheme for elastic properties of rocks with dry or saturated cracks. Geophysical Journal International 151, 597-611.

Brisard, S., Dormieux, L., 2010. FFT-based methods for the mechanics of composites: A general variational framework. Computational Materials Science 49, $663-671$

Brisard, S., Dormieux, L., 2012. Combining galerkin approximation techniques with the principle of hashin and shtrikman to derive a new FFTbased numerical method for the homogenization of composites. Computer Methods in Applied Mechanics and Engineering 217-220, 197 212.

Budiansky, B., 1965. On the elastic moduli of some heterogeneous materials. Journal of the Mechanics and Physics of Solids $13,223-227$.

Cadiou, F., Etiemble, A., Douillard, T., Willot, F., Valentin, O., Badot, J.C., Lestriez, B., Maire, E., 2019. Numerical prediction of multiscale electronic conductivity of lithium-ion battery positive electrodes. Journal of The Electrochemical Society 166, A1692-A1703.

Cai, X., Brenner, R., Peralta, L., Olivier, C., Gouttenoire, P.J., Chappard, C., Peyrin, F., Cassereau, D., Laugier, P., Grimal, Q., 2019. Homogenization of cortical bone reveals that the organization and shape of pores marginally affect elasticity. Journal of The Royal Society Interface 16, 20180911.

Cule, D., Torquato, S., 1999. Generating random media from limited microstructural information via stochastic optimization. Journal of Applied Physics 86, 3428-3437.

Debye, P., Anderson, H.R., Brumberger, H., 1957. Scattering by an Inhomogeneous Solid. II. The Correlation Function and Its Application. Journal of Applied Physics 28, 679-683.

Dirrenberger, J., Forest, S., Jeulin, D., 2013. Effective elastic properties of auxetic microstructures: anisotropy and structural applications. International Journal of Mechanics and Materials in Design 9, 21-33.

Donev, A., Torquato, S., Stillinger, F.H., 2005a. Neighbor list collision-driven molecular dynamics simulation for nonspherical hard particles. I. Algorithmic details. Journal of Computational Physics 202, 737-764.

Donev, A., Torquato, S., Stillinger, F.H., 2005b. Neighbor list collision-driven molecular dynamics simulation for nonspherical hard particles.: II. Applications to ellipses and ellipsoids. Journal of Computational Physics 202, 765-793.

Drummy, L.F., Wang, Y.C., Schoenmakers, R., May, K., Jackson, M., Koerner, H., Farmer, B.L., Mauryama, B., Vaia, R.A., 2008. Morphology of Layered Silicate- (NanoClay-) Polymer Nanocomposites by Electron Tomography and Small-Angle X-ray Scattering. Macromolecules 41, 2135-2143.

Escoda, J., Jeulin, D., Willot, F., Toulemonde, C., 2015. Three-dimensional morphological modelling of concrete using multiscale Poisson polyhedra. Journal of Microscopy 258, 31-48.

Escoda, J., Willot, F., Jeulin, D., Sanahuja, J., Toulemonde, C., 2011. Estimation of local stresses and elastic properties of a mortar sample by FFT computation of fields on a 3d image. Cement and Concrete Research 41, $542-556$.

Eshelby, J., 1957. The determination of elastic field of an ellipsoidal inclusion and related problems. Proceedings of the Royal Society of London , 379-396.

Gatt, J.M., Monerie, Y., Laux, D., Baron, D., 2005. Elastic behavior of porous ceramics: application to nuclear fuel materials. Journal of Nuclear Materials 336, $145-155$

Hashin, Z., Shtrikman, S., 1963. A variational approach to the theory of the elastic behaviour of multiphase materials. Journal of the Mechanics and Physics of Solids, 127-140.

Kanaun, S., 2016. Efficient homogenization techniques for elastic composites: Maxwell scheme vs. effective field method. International Journal of Engineering Science 103, 19-34.

Karsanina, M.V., Gerke, K.M., Skvortsova, E.B., Mallants, D., 2015. Universal Spatial Correlation Functions for Describing and Reconstructing Soil Microstructure. PLOS ONE 10, e0126515.

Khristenko, U., Constantinescu, A., Tallec, P.L., Oden, J.T., Wohlmuth, B., 2020. A statistical framework for generating microstructures of two-phase random materials: Application to fatigue analysis. Multiscale Modeling \& Simulation 18, 21-43.

Korringa, J., 1973. Theory of elastic constants of heterogeneous media. Journal of Mathematical Physics 14, 509-513.

Kröner, E., 1974. On the Physics and Mathematics of Self-Stresses, in: Zeman, J.L., Ziegler, F. (Eds.), Topics in Applied Continuum Mechanics, Springer Verlag Wien, Vienna. pp. 22-38.

Kumar, S., Tan, S., Zheng, L., Kochmann, D.M., 2020. Inverse-designed spinodoid metamaterials. npj Computational Materials 6, 1-10.

Lantuejoul, C., 2002. Geostatistical Simulations: Models and Algorithms. Springer, Berlin/Heidelberg, Germany/New York.

Levitz, P., 1998. Off-lattice reconstruction of porous media: critical evaluation, geometrical confinement and molecular transport. Advances in Colloid and Interface Science 76-77, 71-106.

Liu, Y., Li, J., Sun, S., Yu, B., 2019. Advances in gaussian random field generation: a review. Computational Geosciences . 
Lopez-Pamies, O., Goudarzi, T., Danas, K., 2013. The nonlinear elastic response of suspensions of rigid inclusions in rubber: Ii-a simple explicit approximation for finite-concentration suspensions. Journal of the Mechanics and Physics of Solids 61, 19 - 37.

Michel, J.C., Moulinec, H., Suquet, P., 2001. A computational scheme for linear and non-linear composites with arbitrary phase contrast. International Journal for Numerical Methods in Engineering 52, 139-160.

Milton, G., 1985. The coherent potential approximation is a realizable effective medium scheme. communications in mathematical physics, 99 , 463-500. Communications in Mathematical Physics 99.

Mori, T., Tanaka, K., 1973. Average stress in matrix and average elastic energy of materials with misfitting inclusions. Acta Metallurgica et Materiallia , 571-574.

Morin, L., Gilormini, P., Derrien, K., 2020. Generalized Euclidean Distances for Elasticity Tensors. Journal of Elasticity 138, $221-232$.

Moulinec, H., Suquet, P., 1994. A fast numerical method for computing the linear and nonlinear mechanical properties of composites. Comptes rendus de l'Académie des sciences. Série II, Mécanique, physique, chimie, astronomie .

Moulinec, H., Suquet, P., 1998. A numerical method for computing the overall response of nonlinear composites with complex microstructure. Computer Methods in Applied Mechanics and Engineering 157, 69-94.

Neumann, M., Osenberg, M., Hilger, A., Franzen, D., Turek, T., Manke, I., Schmidt, V., 2019. On a pluri-gaussian model for three-phase microstructures, with applications to 3d image data of gas-diffusion electrodes. Computational Materials Science $156,325-331$.

Neumann, M., Stenzel, O., Willot, F., Holzer, L., Schmidt, V., 2020. Quantifying the influence of microstructure on effective conductivity and permeability: Virtual materials testing. International Journal of Solids and Structures 184, 211-220.

Nirmalraj, P.N., Bellew, A.T., Bell, A.P., Fairfield, J.A., McCarthy, E.K., O’Kelly, C., Pereira, L.F.C., Sorel, S., Morosan, D., Coleman, J.N., Ferreira, M.S., Boland, J.J., 2012. Manipulating Connectivity and Electrical Conductivity in Metallic Nanowire Networks. Nano Letters 12, 5966-5971.

Norris, A., 1985. A differential scheme for the effective moduli of composites. Mechanics of Materials 4, $1-16$.

Øren, P.E., Bakke, S., 2002. Process based reconstruction of sandstones and prediction of transport properties. Transport in Porous Media 46, 311-343.

Ostoja-Starzewski, M., 2006. Material spatial randomness: From statistical to representative volume element. Probabilistic Engineering Mechanics $21,112-132$.

Poirion, F., Soize, C., 1995. Numerical methods and mathematical aspects for simulation of homogeneous and non homogeneous gaussian vector fields, in: Krée, P., Wedig, W. (Eds.), Probabilistic Methods in Applied Physics. Springer Berlin Heidelberg. volume 451 of Lecture Notes in Physics, pp. 17-53.

Ponte Castañeda, P., Willis, J., 1995. The effect of spatial distribution on the effective behavior of composite materials and cracked media. Journal of the Mechanics and Physics of Solids 43, 1919 - 1951.

Ramtani, S., Bui, H.Q., Dirras, G., 2009. A revisited generalized self-consistent polycrystal model following an incremental small strain formulation and including grain-size distribution effect. International Journal of Engineering Science 47, 537-553.

Ren, X., Shen, J., Ghaedizadeh, A., Tian, H., Xie, Y.M., 2015. Experiments and parametric studies on 3d metallic auxetic metamaterials with tuneable mechanical properties. Smart Materials and Structures 24, 095016.

Roberts, A.P., 1997. Statistical reconstruction of three-dimensional porous media from two-dimensional images. Phys. Rev. E 56, $3203-3212$.

Roberts, A.P., Teubner, M., 1995. Transport properties of heterogeneous materials derived from gaussian random fields: Bounds and simulation. Physical Review E 51, 4141-4154.

Roubin, E., Colliat, J.B., 2016. Critical probability of percolation over bounded region in n-dimensional euclidean space. Journal of Statistical Mechanics: Theory and Experiment 2016, 033306.

Roubin, E., Colliat, J.B., Benkemoun, N., 2015. Meso-scale modeling of concrete: A morphological description based on excursion sets of random fields. Computational Materials Science 102, 183 - 195.

Sevostianova, E., Leinauer, B., Sevostianov, I., 2010. Quantitative characterization of the microstructure of a porous material in the context of tortuosity. International Journal of Engineering Science 48, 1693-1701.

Shinozuka, M., Deodatis, G., 1991. Simulation of Stochastic Processes by Spectral Representation. Applied Mechanics Reviews 44, 191-204.

Shinozuka, M., Deodatis, G., 1996. Simulation of Multi-Dimensional Gaussian Stochastic Fields by Spectral Representation. Applied Mechanics Reviews 49, 29-53.

Stenzel, O., Pecho, O., Holzer, L., Neumann, M., Schmidt, V., 2016. Predicting effective conductivities based on geometric microstructure characteristics. AIChE Journal 62, 1834-1843.

Tarantino, M., Zerhouni, O., Danas, K., 2019. Random 3d-printed isotropic composites with high volume fraction of pore-like polydisperse inclusions and near-optimal elastic stiffness. Acta Materialia 175, $331-340$.

Teubner, M., 1991. Level surfaces of gaussian random fields and microemulsions. Europhysics Letters (EPL) 14, 403-408.

Tomita, H., Murakami, C., 1988. Statistics of Random Pattern - Curvature, Percolation and Others, in: Komura, S., Furukawa, H. (Eds.), Dynamics of Ordering Processes in Condensed Matter. Springer US, Boston, MA, pp. 167-172.

Torquato, S., 1997. Effective stiffness tensor of composite media-i. exact series expansions. Journal of the Mechanics and Physics of Solids 45, $1421-1448$.

Torquato, S., 1998. Effective stiffness tensor of composite media : II. Applications to isotropic dispersions. Journal of the Mechanics and Physics of Solids 46, 1411-1440.

Torquato, S., 2002. Random Heterogeneous Materials: Microstructure and Macroscopic Properties. Springer, New York.

Torquato, S., Lado, F., 1986. Effective properties of two-phase disordered composite media: II. Evaluation of bounds on the conductivity and bulk modulus of dispersions of impenetrable spheres. Physical Review B 33, 6428-6435.

Walpole, L.J., 1984. Fourth-Rank Tensors of the Thirty-Two Crystal Classes: Multiplication Tables. Proceedings of the Royal Society of London. A. Mathematical and Physical Sciences 391, 149-179.

Willis, J., 1977. Bounds and self-consistent estimates for the overall properties of anisotropic composites. Journal of the Mechanics and Physics of Solids 25, $185-202$.

Willis, J., 1981. Variational and related methods for the overall properties of composites, Elsevier. volume 21 of Advances in Applied Mechanics, 
pp. $1-78$.

Willot, F., 2015. Fourier-based schemes for computing the mechanical response of composites with accurate local fields. Comptes Rendus Mécanique 343, $232-245$.

Willot, F., Jeulin, D., 2009. Elastic behavior of composites containing Boolean random sets of inhomogeneities. International Journal of Engineering Science 47, 313-324.

Zeller, R., Dederichs, P.H., 1973. Elastic Constants of Polycrystals. Physica Status Solidi (B) 55, 831-842.

Zerhouni, O., Tarantino, M., Danas, K., 2019. Numerically-aided 3d printed random isotropic porous materials approaching the hashin-shtrikman bounds. Composites Part B: Engineering 156, $344-354$. 